DESY $12-192$

HU-EP- $12 / 32$

SFB $/$ CPP- $12-80$

FTUAM-12-99

IFT-UAM/CSIC-12-67

\title{
Overlap valence quarks on a twisted mass sea: a case study for mixed action Lattice QCD
}

\author{
Krzysztof Cichy ${ }^{1,2}$, Vincent Drach ${ }^{1}$, Elena García-Ramos ${ }^{1,3}$, \\ Gregorio Herdoíza ${ }^{4}$, Karl Jansen ${ }^{1}$ \\ ${ }^{1}$ NIC, DESY, Platanenallee 6, D-15738 Zeuthen, Germany \\ ${ }^{2}$ Adam Mickiewicz University, Faculty of Physics, \\ Umultowska 85, 61-614 Poznan, Poland \\ ${ }^{3}$ Humboldt Universität zu Berlin, Newtonstr. 15, 12489 Berlin \\ ${ }^{4}$ Departamento de Física Teórica and Instituto de Física Teórica \\ UAM/CSIC Universidad Autónoma de Madrid, \\ Cantoblanco E-28049 Madrid, Spain

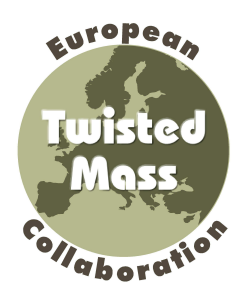

\begin{abstract}
We discuss a Lattice QCD mixed action investigation employing Wilson maximally twisted mass sea and overlap valence fermions. Using four values of the lattice spacing, we demonstrate that the overlap Dirac operator assumes a point-like locality in the continuum limit. We also show that by adopting suitable matching conditions for the sea and valence theories a consistent continuum limit for the pion decay constant and light baryon masses can be obtained. Finally, we confront results for sea-valence mixed meson masses and the valence scalar correlator with corresponding expressions of chiral perturbation theory. This allows us to extract low energy constants of mixed action chiral perturbation which characterize the strength of unitarity violations in our mixed action setup.
\end{abstract}

PACS numbers: 11.15.Ha, 12.38.Gc 


\section{Introduction}

The discovery that despite the no-go theorem of Ref. [1, an exact chiral symmetry can be established for lattice regulated field theories without violating essential field theoretical conditions [2, is certainly one of the major conceptual breakthroughs within lattice field theory in the last years. The fundamental theoretical development for this achievement is the Ginsparg-Wilson relation [3]. The theoretical and conceptual advantages of a lattice Dirac operator obeying this relation have been pointed out in Refs. [4, 5, 6, 7]. A practical solution - suitable for numerical simulations - of an operator satisfying the Ginsparg-Wilson relation, termed overlap Neuberger-Dirac operator, was found in Refs. [8, 9].

Unfortunately, soon after this exciting development in lattice field theory, it turned out that for numerical simulations overlap fermions are very expensive. In fact, the numerical cost is so demanding that many groups working in lattice QCD are nowadays still using lattice discretizations based on computationally much cheaper Wilson or staggered like fermions 1 . Although with such much simpler lattice fermions, presently, simulations very close to or even at the physical value of the pion mass can be performed, see e.g. Ref. [13], it would be nevertheless highly desirable to take advantage of the conceptually much cleaner overlap fermions, or other Ginsparg-Wilson fermions that can be applied in practice, to investigate problems where chiral symmetry or topological aspects play a significant role. Given the high numerical demand of overlap fermions, such simulations are, however, restricted so far to the future, when supercomputers of much higher performance than available today, in combination with, hopefully, much improved algorithms are developed.

An intermediate step towards the goal to employ overlap fermions is to use them only in the valence quark sector, while generating gauge field configurations with much cheaper sea quarks, e.g. Wilson or staggered like quarks. Such a situation is called mixed action setup. The basic idea in such a setup is to relate the sea and valence quark actions by a suitable matching condition, e.g. by demanding that at a given value of the lattice spacing the pion mass is the same in both discretizations. With such a condition, in principle, the continuum limit of a mixed action setup is well defined and should give correct answers for all physical observables of interest.

However, at any non-zero value of the lattice spacing care has to be taken when using mixed actions. Since the sea and valence actions are different,

\footnotetext{
${ }^{1}$ See, however, the work in a chiral invariant Higgs-Yukawa model, where extensive use of the overlap operator is made [10, 11, 12.
} 
there are unitarity violations the size of which is unknown a priori and should be determined. Also, the eigenvalue spectra of the sea and valence lattice Dirac operators are not matched, which can lead to problems especially for the zero mode contributions of the chiral invariant overlap Dirac operator. In addition, the locality property of the overlap Dirac operator [14 needs to be determined in order to see whether hadronic quantities could be affected by finite values of the exponential decay rate. All these aspects demand therefore a principal investigation of a mixed action before large scale simulations can be started.

In this paper, we want to report on one such basic mixed action analysis, 2 , namely using maximally twisted mass fermions 25] in the sea sector and overlap fermions in the valence sector. In a previous work [26, 27], a first study of this mixed action setup has been performed and particular difficulties with such an approach have been reported, which arise from the fact that the exact zero modes of the overlap valence Dirac operator are not matched exactly by the sea quark twisted mass Dirac operator. This phenomenon appears at typical values of the lattice spacing currently used in simulations, i.e. $0.04 \mathrm{fm} \lesssim a \lesssim 0.1 \mathrm{fm}$. In such a situation, special care must be taken, in particular in the way the sea and valence quark theories are matched.

Here we want to extend the analysis of Ref. [27] in several directions. The first is that we add a new and finer value of the lattice spacing than available in Ref. 27] to the analysis of the continuum limit scaling test of the pion decay constant. As we will discuss below, the results of the analysis with this new value of the lattice spacing confirm the findings of Ref. [27] and strengthen the conclusion that there are critical values for the pion mass and the physical volume below which simulations can strongly suffer from the effects of the mismatch of the zero modes between sea and valence sectors. We will also demonstrate that the improved matching condition, suggested in Ref. [27], indeed works well for the continuum limit of the pion decay constant and the nucleon and $\Delta$ masses which are new observables added to the study of the properties of the mixed action we are considering here.

As a second aspect, we want to show that the overlap Dirac operator, which is only exponentially localized at non-zero lattice spacing [14], reaches the expected point-like locality property in the continuum limit. We will use four values of the lattice spacing to perform such a continuum limit extrapolation and, in particular, compare the localization range of the overlap

\footnotetext{
${ }^{2}$ Mixed actions have been used by various groups. Here we mention studies using domain wall sea and overlap valence fermions [15, 16], Wilson clover sea and overlap valence quarks [17, 18, domain wall valence fermions on a staggered sea [19, 20, 21]. Moreover, another class of mixed actions involves using variants of the sea action in the valence sector, such as Osterwalder-Seiler quarks on a twisted mass sea [22, 23, 24].
} 
Dirac operator with hadronic scales, i.e. the pion and the nucleon masses. To our knowledge, this is the first unquenched continuum limit study of the locality properties of the overlap Dirac operator.

As a third and also new investigation, we study the meson masses constructed in the mixed action theory and confront them with expressions of mixed action chiral perturbation theory. This allows us to compute a number of low energy constants of the mixed action effective chiral Lagrangian. In addition, we will look at the non-singlet scalar correlator to test for possible unitarity violation which are inherent in mixed action simulations, as mentioned above.

The main goal of the paper is to provide a basic and principal investigation of a mixed action employing overlap fermions in the valence sector. The results of this investigation can and will help any further calculation aiming at computing physical quantities in that it provides important information on the properties of mixed actions which can help to perform safe simulations in the future.

The outline of the paper is as follows. Sec. 2 discusses some theoretical aspects of our setup and gives parameters of our lattices. In Sec. 3, we look at the continuum limit behaviour of the locality of the overlap Dirac operator. Sec. 4 reports the results of our new and finest lattice spacing by including them in a continuum limit scaling test of the pion decay constant. In Sec. 5, we discuss mixed meson masses and unitarity violations in the non-singlet scalar correlator. In Sec. 6, we employ our mixed action setup in the baryon sector and perform a continuum limit scaling test for nucleon and $\Delta$ masses. Sec. 7 concludes and summarizes our main findings.

\section{Simulation setup}

\subsection{Overlap Fermions}

The fundamental Nielsen-Ninomiya theorem [1, 28], formulated in 1981, apparently excluded the possibility of simulating chiral fermions on the lattice, without violating essential properties, such as the absence of doubler modes or the locality of the theory. However, already in 1982 it was shown by Ginsparg and Wilson [3] that a remnant of chiral symmetry is present on the lattice without the doublers, if the corresponding lattice Dirac operator $\hat{D}$ obeys an equation now called the Ginsparg-Wilson relation:

$$
\gamma_{5} \hat{D}+\hat{D} \gamma_{5}=a \hat{D} \gamma_{5} \hat{D}
$$

For many years, though, no practical solution to this equation had been known. The situation changed in the second part of the 1990s when the 
Ginsparg-Wilson relation was reconsidered and its conceptional advantages pointed out [4, 6, 8, 9]. Moreover, in Ref. [2] the key observation was made that any lattice Dirac operator satisfying the Ginsparg-Wilson relation leads to an exact chiral symmetry at non-vanishing value of the lattice spacing. Neuberger found a particular closed form of a lattice Dirac operator $D-$ called overlap Dirac operator - that obeys the Ginsparg-Wilson relation and that can be employed in practical simulations. The massless overlap Dirac operator is given by $3^{3}[\underline{8}, 9$ :

$$
\hat{D}_{\text {ov }}(0)=\frac{1}{a}\left(1-A\left(A^{\dagger} A\right)^{-1 / 2}\right) .
$$

In the kernel operator $A$ we choose the standard Wilson-Dirac operator:

$$
A=1+s-a \hat{D}_{\mathrm{Wilson}}(0),
$$

where $s$ is a parameter which satisfies $|s|<1$ and can be tuned to optimize locality properties [14] (see Sec. 3 for a detailed test of locality in our setup). The Wilson-Dirac operator is defined by:

$$
\hat{D}_{\text {Wilson }}\left(m_{0}\right)=\frac{1}{2}\left(\gamma_{\mu}\left(\nabla_{\mu}^{*}+\nabla_{\mu}\right)-a \nabla_{\mu}^{*} \nabla_{\mu}\right)+m_{0},
$$

where $m_{0}$ is the bare Wilson quark mass and $\nabla_{\mu}\left(\nabla_{\mu}^{*}\right)$ are the forward (backward) covariant derivatives. The massive overlap Dirac operator with the bare overlap quark mass $m_{\mathrm{ov}}$ reads:

$$
\hat{D}_{\mathrm{ov}}\left(m_{\mathrm{ov}}\right)=\left(1-\frac{a m_{\mathrm{ov}}}{2}\right) \hat{D}_{\mathrm{ov}}(0)+m_{\mathrm{ov}} .
$$

The most important property of overlap fermions is the fact that chiral symmetry in a lattice modified form can be established with very important consequences, among them the absence of $\mathcal{O}(a)$ lattice artefacts.

From the technical side, an important feature of overlap fermions is that the explicit construction of the overlap Dirac operator involves an approximation (in our case the Chebyshev polynomial approximation) of the operator $\left(A^{\dagger} A\right)^{-1 / 2}$ that needs to be realized up to machine precision, in order to consider the lattice chiral symmetry to be exact. This leads to a large computational cost of overlap fermions, around two orders of magnitude larger than of e.g. Wilson twisted mass fermions. For a comparison of overlap and Wilson twisted mass fermions in terms of computing cost, we refer to [29].

Further problems occur in simulations with dynamical overlap fermions. The overlap Dirac operator develops discontinuities when changing topological sectors and the proposed solutions to alleviate this problem [30, 31, 32, 33]

\footnotetext{
${ }^{3}$ For an early review of the overlap fermions formalism, see e.g. Ref. [7].
} 
lead to a further growth of the computational cost. The discontinuity problem can also be avoided by fixing topology [34, 35, which, however, still needs very computer time expensive simulations and introduces additional $\mathcal{O}(1 / V)$ finite volume effects. The mixed action approach, which uses computationally cheap sea quark simulations on which then valence overlap quarks are evaluated, is another way of avoiding the large computational costs of dynamical overlap simulations. This is the setup considered in this paper. Of course, in this approach exact chiral symmetry is only realized in the valence sector.

Since the pseudoscalar decay constant will play an important role in the following, we discuss this observable here. For overlap fermions, the pseudoscalar decay constant $f_{\pi}^{\text {ov }}$ can be extracted using the PCAC relation and does not require the computation of any renormalization constants (see e.g. Ref. [36]):

$$
f_{\pi}^{\mathrm{ov}}=\frac{2 m_{\mathrm{ov}}}{\left(m_{\pi}^{\mathrm{ov}}\right)^{2}}\left|\langle 0|P| \pi\rangle_{\mathrm{ov}}\right|
$$

where $m_{\pi}^{\text {ov }}$ is the mass of the charged pseudoscalar meson and $\left|\langle 0|P| \pi\rangle_{\text {ov }}\right|$ the matrix element of the pseudoscalar current, both extracted from the twopoint pseudoscalar correlation function $C_{\mathrm{PP}}(t)$, built of two mass-degenerate overlap valence quarks.

\subsection{Wilson Twisted Mass Fermions}

Wilson Twisted Mass (tm) fermions [37] were originally introduced to deal with the problem of unphysically small eigenvalues (zero modes) of the WilsonDirac operator. As pointed out in Ref. [25] an essential advantage of this formulation of lattice QCD is also the possibility to obtain automatic $\mathcal{O}(a)$ improvement, by tuning just one parameter - the bare Wilson quark mass to its critical value. This property has been confirmed in detailed continuum limit scaling studies in the quenched approximation [38] and with two dynamical quarks [39]. What is more, the twisted mass discretization can reduce the effects of explicit chiral symmetry breaking by the Wilson term in the renormalization process. In fact, the problem of operators belonging to different chiral representations can be suppressed or even avoided in this formulation. Among the disadvantages of this formulation are the explicit breaking of parity and isospin symmetry, being, however, $\mathcal{O}\left(a^{2}\right)$ cut-off effects [25, 40, 41, 42, 39, which were observed to be substantial only in the neutral pion mass.

Twisted mass fermions are obtained by adding a chirally rotated mass 
term to the Wilson-Dirac operator in Eq. (4) in the following way:

$$
\hat{D}_{\mathrm{tm}}=\hat{D}_{\mathrm{Wilson}}\left(m_{0}\right)+i \mu \gamma_{5} \tau_{3}
$$

where $\mu$ is the twisted mass parameter and $\tau_{3}$ is the third Pauli matrix acting in flavour space.

A comprehensive simulation programme has been undertaken by the European Twisted Mass (ETM) collaboration, including simulations with dynamical up and down quarks $\left(N_{\mathrm{f}}=2\right)$ [43, 44, 39] and with dynamical up, down, strange and charm quarks $\left(N_{\mathrm{f}}=2+1+1\right)$ [45, 46].

The charged pseudoscalar meson decay constant can be obtained in a similar way as in the case of overlap fermions, without the need to compute any renormalization factors:

$$
f_{\pi}^{\mathrm{tm}}=\frac{2 \mu}{\left(m_{\pi}^{\mathrm{tm}}\right)^{2}}\left|\left\langle 0|P| \pi^{ \pm}\right\rangle_{\mathrm{tm}}\right|
$$

where $m_{\pi}^{\mathrm{tm}}$ is the pseudoscalar meson mass and $\left|\left\langle 0|P| \pi^{ \pm}\right\rangle_{\mathrm{tm}}\right|$ the matrix element of the pseudoscalar current, both extracted from the two-point pseudoscalar correlation function $C_{\mathrm{PP}}(t)$, built of two mass-degenerate tm valence quarks.

\subsection{Mixed action setup}

Our mixed action setup consists of two mass-degenerate flavours of Wilson twisted mass quarks at maximal twist in the sea sector and overlap valence fermions. The parameters of our dynamical ensembles are provided in Tab. 1. The ensembles were generated by the ETM Collaboration [39], using the tree-level Symanzik improved gauge action and $N_{\mathrm{f}}=2$ flavours of twisted mass fermions, tuned to maximal twist by setting the hopping parameter $\kappa=1 /\left(8+2 a m_{0}\right)$ to its critical value, at which the PCAC quark mass vanishes [43].

The light-quark ensembles, labeled in Tab. 1] with a subscript $\ell$, correspond to a fixed physical situation with roughly fixed pseudoscalar meson mass $m_{\pi} r_{0} \approx 0.8$ (where $r_{0}$ is the Sommer parameter [47]) and lattice size $L \approx 1.3 \mathrm{fm}$. The non-perturbatively renormalized [48] quark mass $\mu_{q}^{\overline{\mathrm{MS}}}(\mu=2 \mathrm{GeV}) \approx 20 \mathrm{MeV}$, which in infinite volume gives a pseudoscalar meson mass of around $300 \mathrm{MeV}$. The chirally extrapolated values of the Sommer parameter for our ensembles are $r_{0} / a=5.25(2)$ at $\beta=3.90$, $r_{0} / a=6.61(2)$ at $\beta=4.05$ and $r_{0} / a=8.33(5)$ at $\beta=4.20$, which corresponds to lattice spacings $a \approx 0.079 \mathrm{fm}, a \approx 0.063 \mathrm{fm}$ and $a \approx 0.051 \mathrm{fm}$, 
respectively [39]. At $\beta=4.35$, we only have one sea quark mass available, which yields $r_{0} / a=9.82(4)$ and a lattice spacing $a \approx 0.042 \mathrm{fm}$.

For our investigations of locality (Sec. 3), we use ensembles with $L / a=32$ at: $\beta=3.9, a \mu=0.004$ (ensemble $\left.B_{\ell, 32}\right), \beta=4.05, a \mu=0.003\left(C_{\ell, 32}\right.$ ), $\beta=4.2, a \mu=0.0065$ (with a heavier quark mass, ensemble $D_{h, 32}$ ) and $\beta=4.35, a \mu=0.00175\left(E_{\ell}\right)$. In addition, we use two additional volumes at $\beta=3.9, a \mu=0.004$ (with linear lattice extent of $0.6 \mathrm{fm}\left(B_{\ell, 8}\right)$ and $1.6 \mathrm{fm}$ $\left.\left(B_{\ell, 20}\right)\right)$, to check finite size effects in the context of locality.

We have also used one small-volume ensemble with a heavier quark mass, labeled as $B_{h}$. It corresponds to the same lattice size and lattice spacing as $B_{\ell}$, but the renormalized quark mass is $\mu_{q}^{\overline{\mathrm{MS}}}(\mu=2 \mathrm{GeV}) \approx 40 \mathrm{MeV}$, which corresponds to $m_{\pi} r_{0} \approx 1.0$ and a pseudoscalar meson mass $m_{\pi} \approx 450 \mathrm{MeV}$ in infinite volume.

Finally, to explicitly check whether one is safe against the effects of zero modes of the overlap operator, discussed in Ref. [27], we have also considered an ensemble $B_{s}$ that corresponds to a physical extent of the lattice of $L \approx 2 \mathrm{fm}$ and a rather heavy renormalized quark mass of $\mu_{q}^{\overline{\mathrm{MS}}}(\mu=2 \mathrm{GeV}) \approx 45 \mathrm{MeV}$, which yields $m_{\pi} r_{0} \approx 1.0$ and a pseudoscalar meson mass of $m_{\pi} \approx 480 \mathrm{MeV}$ in infinite volume. For this ensemble, $m_{\pi} L \approx 4.7$ and hence the effects of zero modes should be very much suppressed [27].

Our setup for the overlap fermion valence sector has been described in detail in Ref. [49]. Here we only briefly mention that we performed one iteration of HYP smearing [50] of gauge links to reduce the condition number of $A^{\dagger} A$. To assure the best locality properties, we set $s=0$ in Eq. (3) (for details, see the next section).

We have produced all-to-all overlap and twisted mass propagators for a wide range of quark masses, which allowed us to compute three types of correlation functions: sea-sea (unitary tm), valence-valence (overlap) and valence-sea (mixed overlap-tm).

\section{$3 \quad$ Locality of the overlap operator}

The aim of this section is a comprehensive investigation of the locality of the overlap lattice Dirac operator. We will use ensembles at four values of the lattice spacing, which will allow us to perform a continuum limit study of the effective decay rate of the overlap operator to see whether indeed pointlike locality is recovered in the continuum limit. We will also investigate the influence of the parameter $s$ in Eq. (3), as well as finite size effects, for which we use four different physical volumes. Finally, we will perform a comparison of the decay rate with hadronic scales of the theory, in particular 


\begin{tabular}{lccccccc}
\hline \hline Label & $\beta$ & $(L / a)^{3} \times T / a$ & $a \mu$ & $\kappa_{\text {crit }}$ & $m_{\pi}^{\mathrm{tm}} r_{0}$ & $L / r_{0}$ & $m_{\pi}^{\mathrm{tm}} L$ \\
\hline \hline$B_{\ell}$ & 3.90 & $16^{3} \times 32$ & 0.0040 & 0.160856 & 0.84 & 3.0 & 2.5 \\
$C_{\ell}$ & 4.05 & $20^{3} \times 40$ & 0.0030 & 0.157010 & 0.83 & 3.0 & 2.4 \\
$D_{\ell}$ & 4.20 & $24^{3} \times 48$ & 0.0020 & 0.154073 & 0.82 & 2.9 & 2.4 \\
$E_{\ell}$ & 4.35 & $32^{3} \times 64$ & 0.00175 & 0.151740 & 0.74 & 3.3 & 2.4 \\
\hline$B_{h}$ & 3.90 & $16^{3} \times 32$ & 0.0074 & 0.160856 & 1.03 & 3.0 & 3.1 \\
\hline$B_{s}$ & 3.90 & $24^{3} \times 48$ & 0.0085 & 0.160856 & 1.02 & 4.6 & 4.7 \\
\hline$B_{\ell, 8}$ & 3.90 & $8^{3} \times 16$ & 0.0040 & 0.160856 & - & 1.5 & - \\
$B_{\ell, 20}$ & 3.90 & $20^{3} \times 40$ & 0.0040 & 0.160856 & 0.73 & 3.8 & 2.8 \\
$B_{\ell, 32}$ & 3.90 & $32^{3} \times 64$ & 0.0040 & 0.160856 & 0.70 & 6.1 & 4.3 \\
$C_{\ell, 32}$ & 4.05 & $32^{3} \times 64$ & 0.0030 & 0.157010 & 0.69 & 4.8 & 3.3 \\
$D_{h, 32}$ & 4.20 & $32^{3} \times 64$ & 0.0065 & 0.154073 & 1.10 & 3.8 & 4.2 \\
\hline \hline
\end{tabular}

Table 1: The parameters of dynamical $N_{\mathrm{f}}=2$ maximally twisted mass ensembles used in this work. We give the label, the values of the inverse coupling $\beta$, the lattice volume $L^{3} \times T$, the twisted mass parameter $a \mu$, the critical hopping parameter $\kappa_{\text {crit }}=1 /\left(8+2 a m_{\text {crit }}\right)$, the approximate values of the pseudoscalar meson mass and of the lattice size in units of the Sommer scale $r_{0}$ and the product $m_{\pi}^{\mathrm{tm}} L$. As demonstrated in Ref. [27], the values of $m_{\pi}^{\mathrm{tm}} L$ provide a very good estimator for the the role of the zero modes of the overlap operator: they can be large (if $m_{\pi}^{\text {tm }} L<3$ ), potentially large in some observables (if $3<m_{\pi}^{\mathrm{tm}} L<4$ ) or almost negligibly small (if $m_{\pi}^{\mathrm{tm}} L>4$ ).

the pseudoscalar and the nucleon masses, in order to achieve a quantitative measure for the decay rate relative to physical scales. Such a comparison is clearly of importance: as long as the decay rate is smaller than, say, a hadron mass, computations with the overlap operator are not very useful. If, as expected, the overlap operator converges in the continuum limit to the Dirac operator with point-like locality, there must exist a value of the lattice spacing, where the decay rate is much larger than the hadronic scale. It is therefore one goal of this paper to determine the ratio of the hadronic mass and the decay rate and to determine this ratio at the values of the lattice spacings used here.

In this section, we will closely follow the investigation of the locality property of the overlap operator of Ref. [14]. While for small enough values of the lattice spacing, the locality of the overlap operator can be analytically proven, for coarser values the locality property needs to be determined numerically. In fact, in Ref. [14] it has been demonstrated that the locality property can be maintained to rather coarse values of the lattice spacing of 
about $a \approx 0.1 \mathrm{fm}$, which has been pushed to even coarser values of $a \approx 0.2$ $\mathrm{fm}$ in [51]. Since the values of the lattice spacings employed in this work are all below $0.1 \mathrm{fm}$, we expect that the valence overlap operator is exponentially localized for all ensembles considered, an expectation which will be confirmed below.

\subsection{Effective decay rate}

It is clear that the overlap Dirac operator (denoted $D$ in this section) is not local in a geometrical sense, which would mean that there exists a finite $\xi$ such that the following requirement is fulfilled:

$$
\begin{aligned}
& D \Psi(n)=\sum_{m} D(n, m) \Psi(m), \\
& D(n, m)=0 \text { for all points } n, m \text { such that }\|n-m\|>\xi,
\end{aligned}
$$

for some norm $\|\cdot\|$ to be defined below. However, from the point of view of continuum limit behaviour of physical quantities, such geometrical locality is not necessary. It is sufficient if the Dirac operator $D(n, m)$ decays exponentially fast, i.e.:

$$
\|D(n, m)\| \leq C \mathrm{e}^{-\hat{\rho}\|n-m\|}
$$

with some decay rate $\hat{\rho}$ (in lattice units, with corresponding physical decay rate $\rho=\hat{\rho} / a$ ) and normalization factor $C$.

For the norm in Eq. (9), we use the taxi-driver distance for periodic boundary conditions:

$$
\|n\|_{1}=\sum_{\mu} \min \left\{\left|n_{\mu}\right|,\left|N_{\mu}-n_{\mu}\right|\right\}, \quad 0 \leq n_{\mu}<N_{\mu},
$$

where $N_{\mu}$ is the number of lattice sites in direction $\mu$. We also define the norm of the operator as the row sum norm:

$$
\|D(n, m)\| \equiv \max _{\substack{1 \leq \mu \leq 4 \\ 1 \leq a \leq 3}} \sum_{\nu=1}^{4} \sum_{b=1}^{3}\left|D(n, m)_{a, b}^{\mu, \nu}\right|
$$

where $\mu, \nu$ denote the Lorentz indices and $a, b$ the colour indices. For the same value of the taxi-driver distance, the operator norm can take several values. Hence, we also define the maximum norm:

$$
\|D\|_{\max }(d)=\max _{\|n-m\|_{1}=d}\|D(n-m, 0)\| .
$$

From now on, we will only consider the maximum norm, since it corresponds to the most restrictive case, i.e. proving locality using the maximum norm implies locality for any other choice of the norm. 


\subsection{Continuum limit analysis}

In this subsection, we will perform a continuum limit scaling test of the decay rate of the overlap operator using ensembles $B_{\ell, 32}, C_{\ell, 32}, D_{h, 32}, E_{\ell}$ of Tab. 1 . These ensembles correspond to four lattice spacings, at different physical volumes, but keeping constant the lattice size $L / a=32$.

The Dirac operator is local if it fulfills Eq. (10). We define the effective decay rate $\hat{\rho}_{\text {eff }}$ as follows:

$$
\hat{\rho}_{\text {eff }}=\ln \left(\frac{\|D\|_{\max }\left(n_{i}\right)}{\|D\|_{\max }\left(n_{i}+1\right)}\right) .
$$

Larger values of the decay rate imply better locality properties.

The full decay property of the overlap operator, including small distances, is determined by a sum of exponential decays with corresponding decay rates:

$$
\|D\|_{\max }=\sum_{i} C_{i} \mathrm{e}^{-\rho_{i}\left\|x_{i}\right\|} \stackrel{\|x\| \rightarrow \infty}{\longrightarrow}\|D\|_{\max }=C \mathrm{e}^{-\rho\|x\|}
$$

Therefore, the desired decay rate is only attained at asymptotically large distances, where the effective decay rate $\hat{\rho}_{\text {eff }}$ will be constant as a function of the taxi-driver distance.

In Fig. 1, we show the effective decay rate as a function of the taxi-driver distance for four different values of the lattice spacing. Our typical statistics was around 50 configurations well separated in Monte Carlo time. In order to observe the expected plateau-like behaviour of $\hat{\rho}_{\text {eff }}$, it is necessary to have a large value for $L / a$. If $L / a$ is too small, the signal is dominated by hypercubic artefacts and the extracted values of $\rho$ are not reliable. We have studied this effect in free-field theory, for different values of $L / a$ and we have concluded that $L / a=32$ is necessary to observe a stable plateau of the effective decay rate (see Sec. 3.5 for details).

Moreover, from Fig. 1 it is clear that the determination of the effective decay rate is not straightforward, i.e. the observed large fluctuations have to be taken into account in the analysis as a systematic error. To this end, we follow the strategy of Refs. [39, 52]. As a first step, we fit the effective decay rates obtained at different values of the taxi-driver distance to a constant, including at least 4 consecutive points. For this, we fit the value of the decay rate and the corresponding statistical error using a jackknife procedure. For each fit, we also compute the corresponding value of $\chi^{2}$ and the confidence level CL, defined as:

$$
\mathrm{CL}\left(q=\chi^{2}, n=\text { d.o.f. }\right)=1-\gamma(q / 2, n / 2),
$$



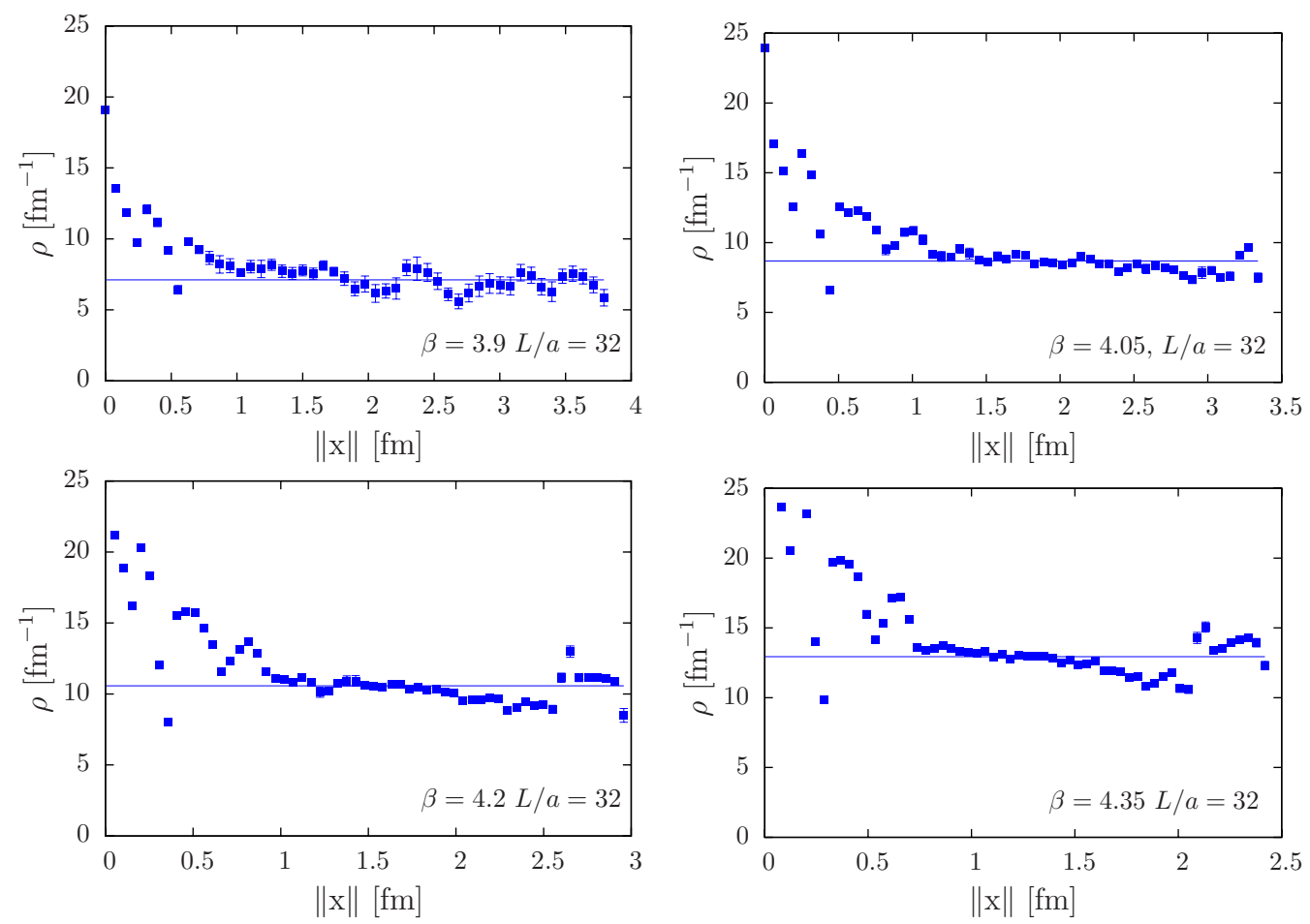

Figure 1: Effective decay rate $\rho\left[\mathrm{fm}^{-1}\right]$ as a function of the taxi driver distance $[\mathrm{fm}]$ for four different values of the lattice spacing. The blue line is the final fit obtained following the method explained in the text. The fitting intervals [fm] are indicated in Tab. 3. The error bars are often smaller than the symbol size; therefore the large fluctuations induce significant systematic uncertainties in the determination of the effective decay rate.

where d.o.f. denotes the number of degrees of freedom for a given fit and $\gamma(q / 2, n / 2)$ is the lower incomplete Gamma function.

In this way, we obtain a large sample of fitted values of the effective decay rate from which we can construct weighted distributions using two kinds of weights, the confidence level (CL) or a function of $\chi^{2}$ which we choose as $\exp \left(\chi^{2}\right) /$ d.o.f. - thus damping the influence of bad fits. From this weighted distribution, we extract the mean and the median of the distribution. In practice, for our final values of the effective decay rates (shaded entry in Tab. 2), we look for the "best fits", i.e. the ones closest to the mean and median of the weighted distribution and satisfying the constraint that their confidence level is at least $80 \%$. The statistical error is then the jackknife error of the best fit, while the systematic error is given by the $68.3 \%$ confidence interval of the weighted distribution. The final error is computed by adding 
both errors in quadrature. We also analyzed the effect of adding a cut in the $\mathrm{x}$ axis in order to avoid possible finite volume effects at large values of the taxi driver distance (cut at $\|x\|=L$ or $\frac{\sqrt{7} L}{2}$, i.e. the maximum distance in a $L^{3} \times(2 L)$ box in the continuum, with periodic boundary conditions), and we found results that are fully compatible with the ones without the cut, however, with a decrease of the systematic error.

Combining all these possibilities to extract the effective decay rate by using the mean and median from the weighted distribution (labeled mean and median in Tab. 2) using either the confidence level or $\chi^{2} /$ d.o.f., mean and median from "best fit" labeled fit(mean) and fit(median) in Tab. 2), we thus have in total 8 different ways of computing the effective decay rate and its statistical 4 and systematic error, see Tab. 2.

In Fig. 2, we show the comparison of the final results for the effective decay rate obtained using these 8 different methods, for the case of the ensemble $E_{\ell}$. All methods lead to compatible results both in the central value, as well as in the total error, which gives us confidence that we indeed have the systematic errors of the effective decay rate under control.

For the continuum limit scaling test of the effective decay rate, we decided to use method 4 of Tab. 2, taking the value for $\rho$ that yields a fitted $\rho$ as close as possible to the median of the CL-weighted histogram. However, we want to emphasize that all other variants would be equally good choices.

Before discussing the numerical results for the effective decay rate, we show in Fig. 3 the exponential decay of the maximum norm of the overlap Dirac operator as a function of the taxi-driver distance. We use four different lattice spacings, ranging from 0.079 to $0.042 \mathrm{fm}$, while keeping the value of $L / a=32$ fixed, as discussed above. We observe that decreasing the lattice spacing leads to an increasing slope of $\left\|D_{\text {ov }}\right\|_{\max }$. This signals the continuum limit restoration of point-like locality.

In Tab. 3, we summarize the results obtained for the values of the decay rate $\rho$, together with their statistical, systematic and total errors. As already suspected from Fig. 1 the total error is dominated by the systematic part.

Using data from Tab. 3, we perform the continuum limit scaling test of the inverse decay rate (locality radius) $1 / \rho$. The values at non-zero lattice spacings can be interpreted as the physical length that correspond to the slowest decrease of the Dirac operator norm. The value of the locality radius extrapolated to $a=0$ is $0.007(9)$ fm, i.e. compatible with zero. Thus, in the continuum limit, point-like locality is indeed restored. If we consider the

\footnotetext{
${ }^{4}$ The statistical error quoted for the values of the mean and the median extracted from the distributions were obtained by analyzing the weighted distribution of all the statistical errors of the different fits computed with jackknife.
} 


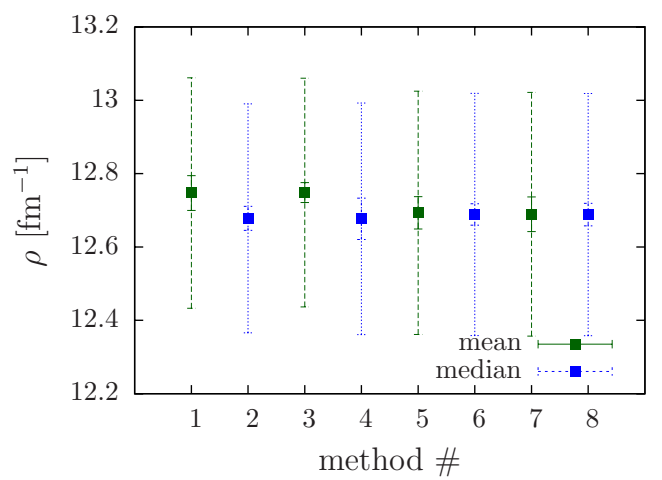

Figure 2: Comparison of results for the ensemble $E_{\ell}$ obtained through different methods of extracting the effective decay rate. The method numbering is explained in Tab. 2, The errors are statistical (smaller error) and systematic (larger error).

\begin{tabular}{ccc}
\hline$\#$ & $\rho$ and $\delta \rho_{\text {stat }}$ & weight \\
\hline 1 & mean & CL \\
\hline 2 & median & CL \\
\hline 3 & fit $($ mean $)$ & CL \\
\hline 4 & fit (median $)$ & CL \\
\hline 5 & mean & $\exp \left(\chi^{2} /\right.$ dof $\left.)\right)$ \\
\hline 6 & median & $\exp \left(\chi^{2} /\right.$ dof $\left.)\right)$ \\
\hline 7 & fit $($ mean $)$ & $\exp \left(\chi^{2} /\right.$ dof $\left.)\right)$ \\
\hline 8 & fit $($ median $)$ & $\exp \left(\chi^{2} /\right.$ dof $\left.)\right)$ \\
\hline
\end{tabular}

Table 2: Different methods used to calculate the value of the effective decay rate $\rho$ and the corresponding error.

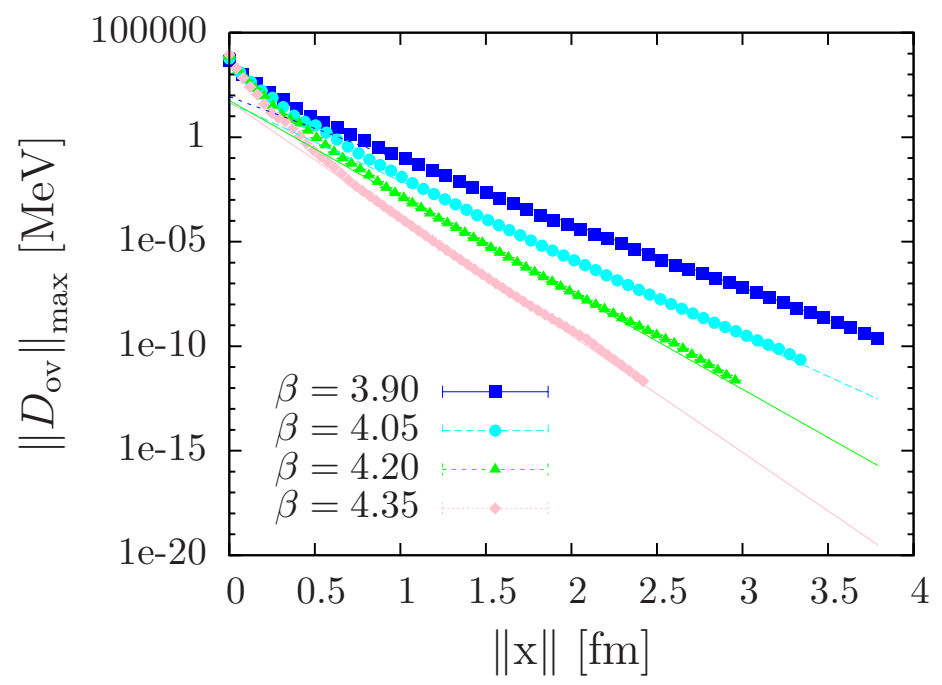

Figure 3: Exponential decay of the maximum norm of the overlap Dirac operator for ensembles $B_{\ell, 32}, C_{\ell, 32}, D_{h, 32}, E_{\ell}$.

lattice artefacts and therefore perform a fit adding a term quadratic in $a$, instead of a simple linear fit, we obtain a compatible result: $-0.002(37) \mathrm{fm}$, but with a much larger error, indicating that we are not sensitive to $\mathcal{O}\left(a^{2}\right)$ cut-off effects in the effective decay rates. 


\begin{tabular}{ccccccc}
\hline & $\begin{array}{c}\rho \\
{\left[\mathrm{fm}^{-1}\right]}\end{array}$ & $\begin{array}{c}\delta_{\text {stat }} \rho \\
{\left[\mathrm{fm}^{-1}\right]}\end{array}$ & $\begin{array}{c}\delta_{\text {syst }} \rho \\
{\left[\mathrm{fm}^{-1}\right]}\end{array}$ & $\begin{array}{c}\delta \rho \\
{\left[\mathrm{fm}^{-1}\right]}\end{array}$ & $\begin{array}{c}1 / \rho \\
{[\mathrm{fm}]}\end{array}$ & $\begin{array}{c}\text { interval } \\
{[\mathrm{fm}]}\end{array}$ \\
\hline \hline$B_{\ell, 32}$ & 7.117 & 0.183 & 0.557 & 0.586 & $0.141(12)$ & {$[2.923,3.634]$} \\
$C_{\ell, 32}$ & 8.684 & 0.065 & 0.333 & 0.339 & $0.115(4)$ & {$[1.953,2.205]$} \\
$D_{h, 32}$ & 10.555 & 0.037 & 0.141 & 0.146 & $0.094(1)$ & {$[1.326,1.785]$} \\
$E_{\ell}$ & 12.677 & 0.056 & 0.311 & 0.316 & $0.079(2)$ & {$[1.176,1.302]$} \\
\hline
\end{tabular}

Table 3: Results for the decay rate $\rho$ and the corresponding errors: statistical $\delta_{\text {stat }} \rho$, systematic $\delta_{\text {syst }} \rho$, total $\delta \rho$. We also give the inverse decay rate (with its combined statistical and systematic error in parentheses) and the fit interval for the best fit.

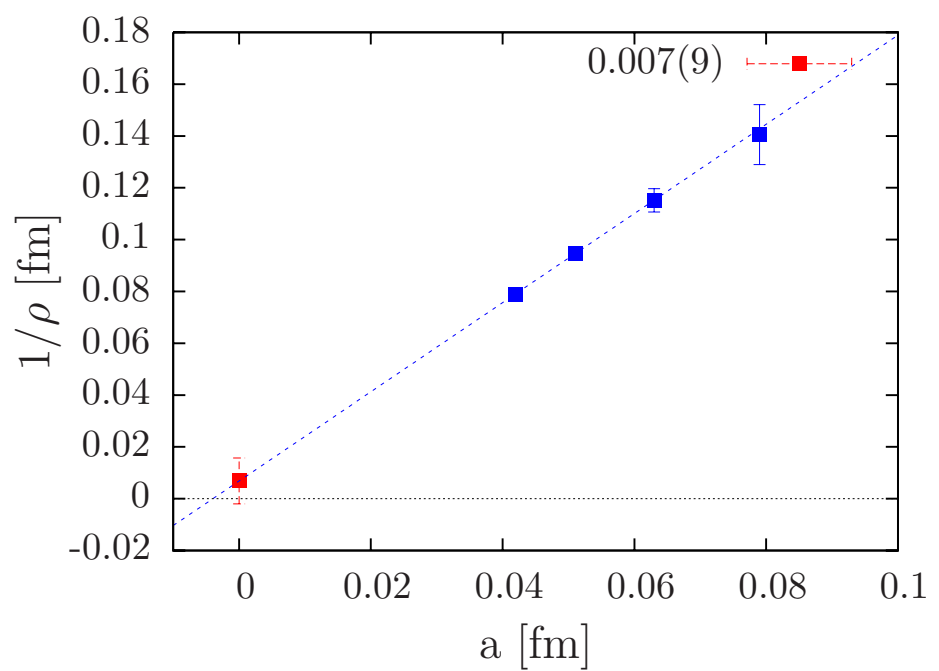

Figure 4: Continuum limit scaling of the inverse decay rate $1 / \rho$.

\subsection{Comparison of decay rates and hadron masses}

The relevance of the decay rate $\rho$ of the overlap operator can be quantified when compared to the size of a hadron. The locality radius, $r_{\text {loc }}=1 / \rho$, is expected to be sufficiently smaller than the hadron size in order to extract the hadron mass without being affected by non-locality problems. To illustrate this point, we consider the approximation in which the size of the hadron is related to the inverse of its mass $m$. There is a similarity of the asymptotic exponential decay of zero-momentum correlation functions (with Euclidean time) and the likewise asymptotic exponential decay of the overlap Dirac operator norm (with taxi-driver distance). At large Euclidean time or taxi- 
driver distance one finds (barring effects from periodic boundary conditions):

$$
\begin{aligned}
& \|D(\|n\|)\|=A \mathrm{e}^{-\hat{\rho}\|n\|} \quad C(t, \mathbf{p}=0)=\tilde{A} \mathrm{e}^{-m t} \\
& \hat{\rho}_{\text {eff }}=\ln \frac{\|D(n)\|}{\|D(n+1)\|} \quad \longleftrightarrow \quad m_{\text {eff }}=\ln \frac{C(t)}{C(t+1)} .
\end{aligned}
$$

where $m$ corresponds to the mass of the lightest hadron with given quantum numbers and $\hat{\rho}_{\text {eff }}$ denotes the effective decay rate.

In this simplified picture, in order to have an extraction of a hadron mass which is not influenced by a possibly too slow decay rate of the overlap operator, the decay rate is expected to be larger than the measured hadron mass: $\hat{\rho}>m a$.

In terms of the Compton wavelength of the hadron $\lambda_{C}=1 / \mathrm{m}$ and the locality radius $r_{\text {loc }}=1 / \rho$ we want $\lambda_{C}>r_{\text {loc }}$. When the condition $\lambda_{C}>r_{\text {loc }}$ is met, once we have reached the asymptotic Euclidean time region to extract masses, the norm of the overlap operator has decayed already sufficiently strongly such as not to influence the hadron mass measurement.

We now show that the condition $\lambda_{C}>r_{\text {loc }}$ is indeed satisfied in our mixed action simulations, considering the masses of the lightest particles in the meson and baryon sectors. We take the unitary values of the pion and nucleon mass, given in Ref. [53. Since we match the pion mass in our mixed action setup, the values of the pion mass are the same as in the unitary setup, by definition, whereas the masses of the nucleon can differ from the unitary ones by $\mathcal{O}\left(a^{2}\right)$ effects. These turn out to be very small in practice - see Sec. 6 for details. We summarize the values for the pion mass, the nucleon mass, the decay rate and the ratios of the masses and the decay rate in Tab. 4.

In Fig. 5, we show the ratio $a m / \hat{\rho}$ as a function of the lattice spacing $a$ in a fixed physical situation, see above. Both for the cases of the pion and the nucleon masses, the values of the ratio are below one (slightly above one in the case of the nucleon at $\beta=3.9$ ) and the continuum limit value is compatible with zero. This suggests that the overlap Dirac operator is sufficiently local in our mixed action setup to allow for a clean computation of these masses. However, working at a coarser lattice spacing or considering heavier hadrons, one could enter the regime where the size of the hadron of interest is smaller than the locality radius. When using overlap fermions, it is thus crucial to monitor that the locality radius is sufficiently smaller than the hadron size for all the values of the lattice spacing which are being considered. 


\begin{tabular}{ccccc}
\hline Ensemble & $a m_{\pi}$ & $a m_{\pi} / \hat{\rho}$ & $a m_{N}$ & $a m_{N} / \hat{\rho}$ \\
\hline$B_{\ell}$ & $0.159(2)$ & $0.283(24)$ & $0.511(6)$ & $1.056(90)$ \\
$C_{\ell}$ & $0.121(4)$ & $0.221(11)$ & $0.409(6)$ & $0.908(41)$ \\
$D_{\ell}$ & $0.098(2)$ & $0.182(4)$ & $0.305(4)$ & $0.756(28)$ \\
$E_{\ell}$ & $0.075(2)$ & $0.141(5)$ & - & - \\
cont.limit & - & $-0.025(21)$ & - & $0.17(16)$ \\
cont.limit (quadratic) & - & $-0.10(9)$ & - & $-0.3(1.2)$ \\
\hline
\end{tabular}

Table 4: The pion masses $m_{\pi}$ and nucleon masses $m_{N}$ in lattice units and the ratios of these masses divided by the decay rate of the overlap Dirac operator $a m_{\pi} / \hat{\rho}$ and $a m_{N} / \hat{\rho}$. The continuum limit values quoted in the table correspond to a linear extrapolation in the first case and in the second case we added a quadratic term to take into account possible higher order lattice artefacts.
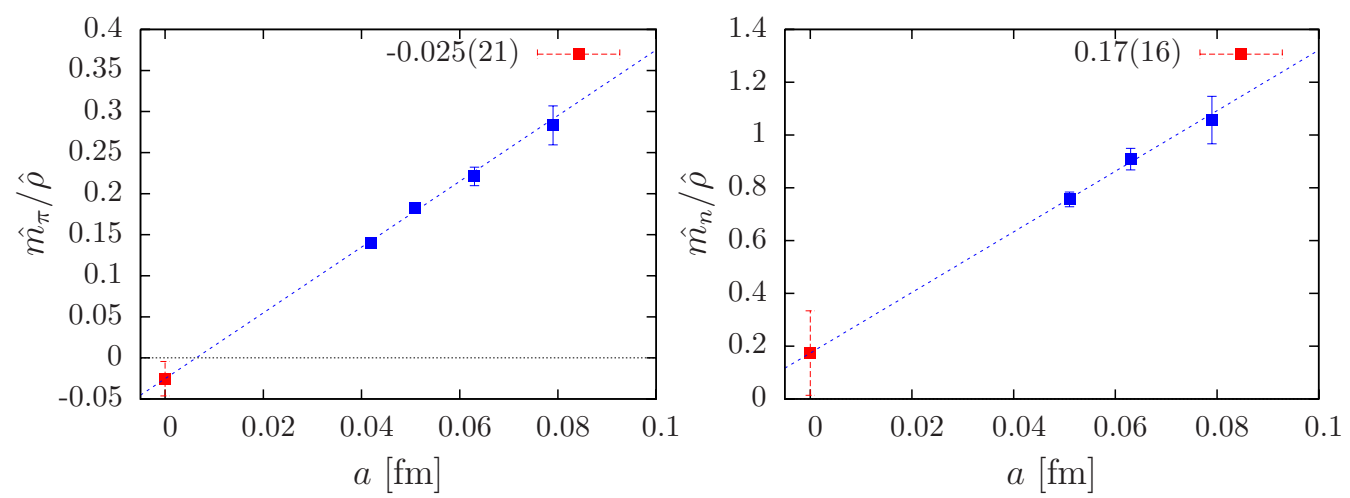

Figure 5: Continuum limit scaling of $a m / \hat{\rho}$ for the pion (left) and nucleon (right) masses. In these plots only the linear fits are shown.

\subsection{Dependence on the $s$ parameter}

The overlap Dirac operator is constructed from the Wilson Dirac operator taken at large negative mass shift, depending on the $s$ parameter (see Eq. (3i)), which has to fulfill $|s|<1$, as shown in Ref. [54]. In this subsection, we analyze the influence of the $s$-parameter on the decay rate $\rho$ of the overlap operator norm. This allows to choose the optimal value of $s$ for simulations, i.e. the one that gives the highest value of $\rho$. We compare the case when our gauge field configurations were HYP smeared (1 iteration) - which is the case for the results in the previous sections - with the one of un-smeared configurations. Since HYP smearing brings the plaquette values closer to 

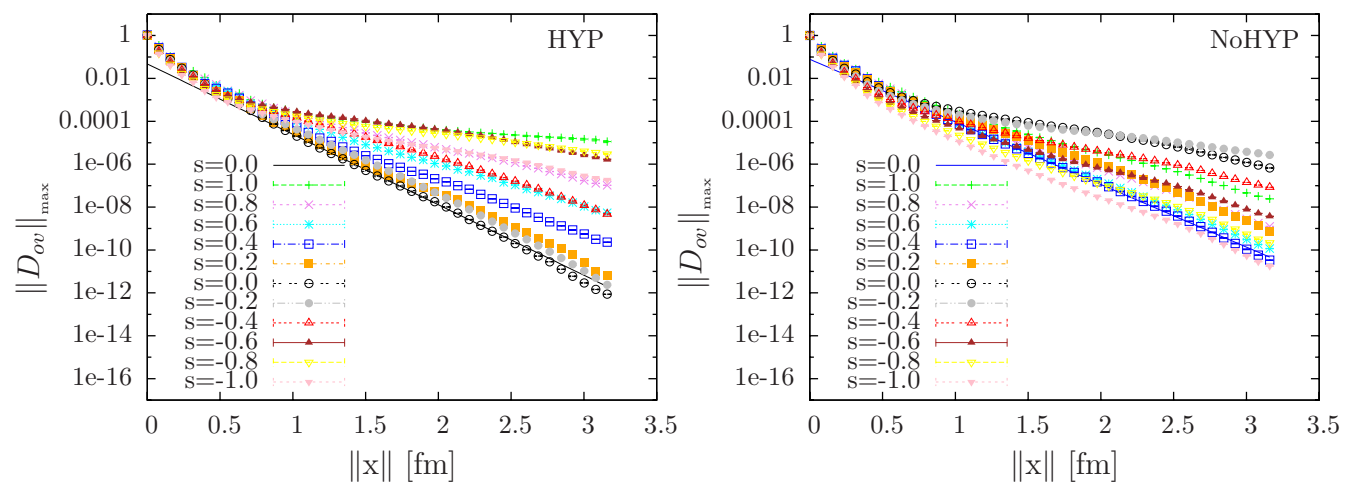

Figure 6: Exponential decay of the norm of the overlap Dirac operator (normalized by the value at $\|x\|=0$ ) for several values of the $s$ parameter as a function of the taxi-driver distance. The graph represents our results for the Ensemble $B_{\ell}$. (left) 1 iteration of HYP smearing was applied to our gauge field configurations. (right) No HYP smearing applied.

unity and lifts the low-lying eigenvalues of the kernel Wilson-Dirac operator, improved locality can be expected and is one of the motivations of using HYP smearing in our mixed action setup.

In Fig. 6, we plot the dependence of the norm of the overlap Dirac operator on the taxi-driver distance, for the ensemble with the coarsest lattice spacing $B_{\ell}$. We compare several values of the $s$ parameter for HYP smeared and original, non-smeared configurations. In all cases, we observe an exponential decay, with a significant dependence on the $s$ parameter. The resulting values of the decay rate $\rho$, which are extracted using the strategy described above, are shown in Fig. 7. For the case of HYP smeared configurations, the optimal value of $s$ is the free-field optimal value $s=0$, in accordance with Ref. [55], while for the original configurations $s=0.4$. In general, we see a strong dependence of $\rho$ on the $s$-parameter and it seems from Fig. 7 that the main difference between HYP-smeared and non-smeared gauge field configurations is solely a shift in the $s$-parameter. In particular, within the errors, there seems to be no clear gain of using smeared or non-smeared configurations for the effective decay rate. Nevertheless, we do observe that in the HYPsmeared case the norm of the Dirac operator $\left\|D_{o v}\right\|$ reaches lower values for the same values of the taxi driver distance than in the Non-HYP smeared case, as is shown in Fig. 6. 


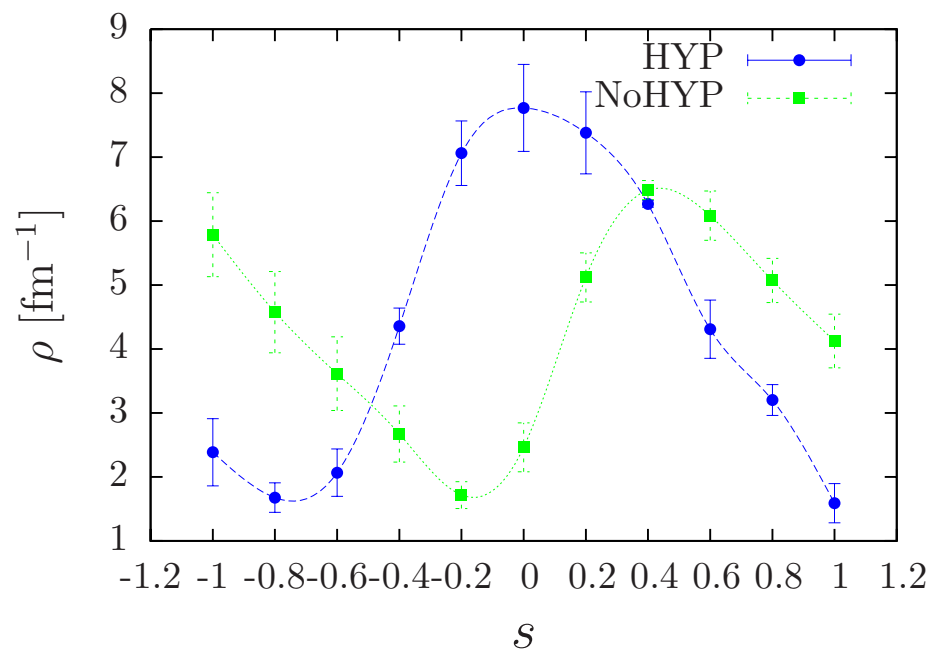

Figure 7: The dependence of the decay rate $\rho$ on the value of the $s$ parameter for HYP and non HYP smeared configurations. The lines are just to guide the eye.

\subsection{Finite Volume Effects}

Since our locality analysis was performed for different physical volumes (from ca. 1.3 to $2.5 \mathrm{fm}$ ), it is natural to ask whether some of these volumes are not too small for a reliable calculation of the decay rate. In order to see whether finite size effects affect the results for the decay rate, we extended our analysis for ensembles $B_{\ell, 32}, C_{\ell, 32}, D_{\ell, 32}$ and $E_{\ell}$ to the corresponding ensembles with smaller physical volumes: $B_{\ell, 8}, B_{\ell}, B_{\ell, 20}, C_{\ell}$ and $D_{\ell}$.

We illustrate in Fig. 8 the outcome of this analysis by comparing results for ensembles $C_{\ell}$ and $C_{\ell, 32}$ (although they are practically the same at other values of $\beta$ ). The upper plots show that in both cases we observe an exponential decay of the overlap Dirac operator norm. However, as the lower plots show, for the case of $L / a=20$, we observe no plateau of the effective decay rate and hence we cannot perform meaningful fits and extract a reliable value of the decay rate5. Further investigation of the problem in free-field theory for several values of $L / a$ indicates the presence of large hypercubic artefacts, reaching taxi-driver distances as large as $\|x\| / a \approx 20$. Moreover, it is natural to expect that at $\|x\| / a \approx L / a$ finite volume effects start to be-

\footnotetext{
${ }^{5}$ If we insist on extracting a value, it is compatible with the one of $L / a=32$, but within a large systematic error - it therefore shows that our way of extracting the systematic error is reliable. We emphasize that even in the case of $L / a=32$, we have to take into account systematic effects, which dominate over statistical errors.
} 

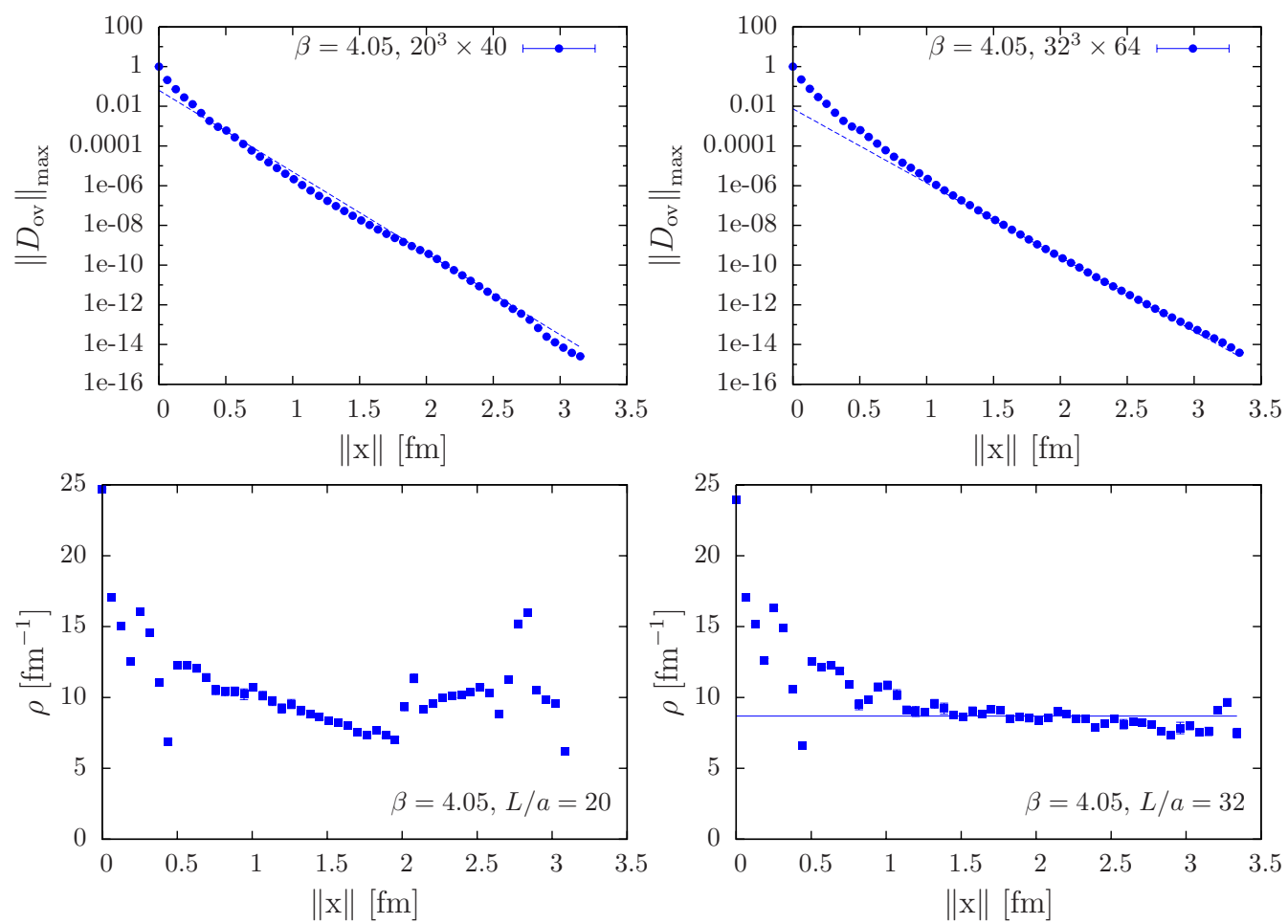

Figure 8: (upper) Exponential decay of the norm of the overlap Dirac operator (normalized by the value at $\|x\|=0$ ) and the effective decay rate $\rho$ (lower) as a function of the taxi driver distance $[\mathrm{fm}]$ for $\beta=4.05, L / a=20$ (left) and $L / a=32$ (right). In the lower left plot, we observe no plateau. In the right plot, the plateau is present and hence we can perform meaningful fits.

come large. Hence, the plateau region of the effective decay rates is located approximately between $\|x\| / a=20$ and 32 , as observed in the lower right plot of Fig. 8. This conclusion is valid for all values of $\beta$, as can be seen in Fig. 1 and it motivated our choice of $L / a=32$ for all lattice spacings, rather than keeping the physical extent $L$ fixed. Indeed, we observe that the decay rate does not depend in a significant way on the volume once the lattice size is sufficiently large. Therefore, for the range of lattice spacings considered in this work, we expect that the finite size effects in the decay rate are negligible when working on $L / a=32$ lattices. We have also checked that performing the continuum limit with the physical volume fixed to 1.3 fm yields a compatible result, but with much worse precision, due to large hypercubic artefacts for $L / a=16(\beta=3.9)$ and $L / a=20(\beta=4.05)$. 


\section{Pion decay constant and the role of the zero modes}

In Ref. [27, we have reported the results of a continuum limit scaling test of the pion decay constant $f_{\pi}$, using maximally twisted mass (MTM) sea fermions and two kinds of valence quarks: MTM (unitary setup) and overlap (mixed action setup). In the mixed action setup, both kinds of fermion actions were matched, using the pion mass or by employing alternative matching conditions. While in the matched mixed action setup the value of $f_{\pi}$ (or any other observable) at finite lattice spacing might be different for two different fermion discretizations, one expects that this difference vanishes in the continuum limit, provided that the matching conditions were chosen properly. Let us shortly summarize the findings of Ref. [27].

In order to compare the continuum limit of $f_{\pi}$ for the unitary and the mixed action setups, we matched them by employing several matching conditions.

1. Naive matching condition - we extracted the pion mass from the correlator constructed from the pseudoscalar interpolating field (PP correlator $\left.C_{P P}(t)\right)$ for both MTM and overlap valence quarks. We defined the matching point as the overlap valence quark mass that yields the same PP correlator pion mass as in the unitary case.

2. Improved matching condition - the unitary pion mass was extracted from the PP correlator, but the overlap valence pion mass was extracted from a correlator defined as $C_{P P-S S}(t)=C_{P P}(t)-C_{S S}(t)$. In such a correlator (PP-SS correlator), the effects of zero modes exactly cancel, since zero modes couple in the same way to the pseudoscalar and scalar correlators. We defined the matching point as the overlap valence quark mass that yields the same PP-SS correlator pion mass as the PP correlator pion mass of the unitary case.

3. Alternative matching condition - matching was performed using the PP correlator, but at a heavier quark mass, using partially quenched MTM data. In this way, one also suppresses the effects of zero modes, since their leading contribution is proportional to the squared inverse quark mass.

For the regime of parameters that we had chosen, we found that using the naive matching condition leads to incompatible continuum limits for $r_{0} f_{\pi}^{\mathrm{tm}}$ and $r_{0} f_{\pi}^{\text {ov }}$, if both are extracted from the PP correlator. This was attributed to the effects of zero modes that couple strongly to this correlator when 


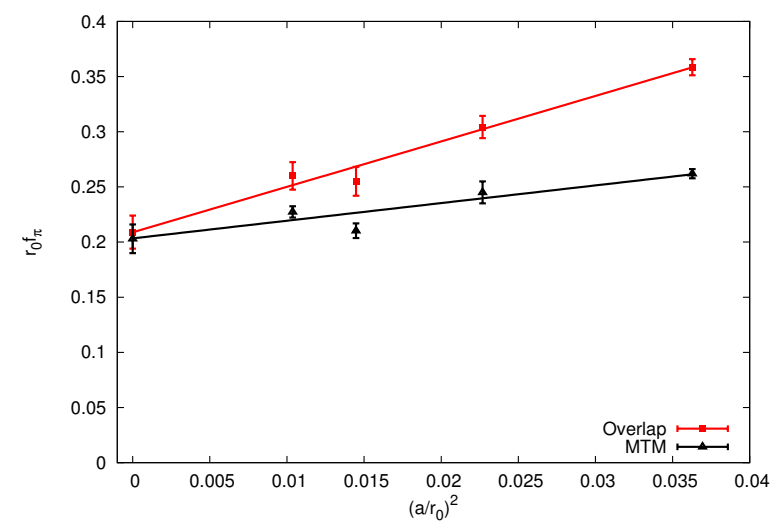

Figure 9: Continuum limit scaling of $r_{0} f_{\pi}$. We used the improved matching condition and extracted $f_{\pi}^{\text {ov }}$ from the PP-SS correlator. The solid line represents a linear extrapolation in $a^{2}$ to the continuum limit.

\begin{tabular}{lcccc}
\hline \hline Ensemble & $a m_{\pi}$ & $\begin{array}{c}a m_{\mathrm{ov}}^{\text {match }} \\
\text { naive }\end{array}$ & $\begin{array}{c}a m_{\mathrm{ov}}^{\text {match }} \\
\text { improved }\end{array}$ & $\begin{array}{c}a m_{\mathrm{ov}}^{\text {match }} \\
\text { alternative }\end{array}$ \\
\hline \hline$B_{\ell}$ & $0.1592(24)$ & 0.007 & 0.011 & 0.009 \\
$C_{\ell}$ & $0.1209(40)$ & 0.005 & 0.006 & 0.006 \\
$D_{\ell}$ & $0.0980(19)$ & 0.002 & 0.004 & 0.004 \\
$E_{\ell}$ & $0.0749(16)$ & 0.004 & 0.004 & - \\
\hline \hline
\end{tabular}

Table 5: The pseudoscalar masses and overlap matching quark masses for different ensembles and different matching conditions. Results for ensembles $B_{\ell}, C_{\ell}$ and $D_{\ell}$ are taken from Ref. [27]. The ensemble $E_{\ell}$ at a very fine value of the lattice spacing has been newly analyzed in this work.

overlap valence quarks are used. These zero mode contributions are not matched by the MTM sea quark action, at least not at the values of the lattice spacing used in Ref. [27]. To test this hypothesis, we employed the improved matching condition and extracted $f_{\pi}^{\text {ov }}$ from the PP-SS correlator. In this way, we found compatible continuum limits. By investigating the three matching conditions described above, we finally concluded that each of them can lead to compatible continuum limits of $f_{\pi}^{\text {tm }}$ and $f_{\pi}^{\text {ov }}$, provided that the latter is extracted from the PP-SS correlator, which does not have the contribution of zero modes.

The aim of the remainder of this subsection is to update this analysis with the fourth lattice spacing (ensemble $E_{\ell}$ at $\beta=4.35$ ). We followed the 
same procedure as in the case of the coarser lattice spacing ensembles $\left(B_{\ell}\right.$, $\left.C_{\ell}, D_{\ell}\right)$ - we extracted the quark mass dependence of the overlap pion mass from the PP-SS correlator and found the matching mass, employing thus the improved matching condition. The values of the matching mass, employing different matching conditions are summarized in Tab. 5. At this matching mass, we computed the pion decay constant $f_{\pi}^{\text {ov }}$ from Eq. (66) and compared it to the unitary value $f_{\pi}^{\mathrm{tm}}$, computed from Eq. (8). The results for our 4 lattice spacings are presented in Fig. 9.

We obtained a result which is fully consistent with our finding in Ref. [27]. Both the unitary setup and the mixed action setup lead to compatible continuum limits (compatible between themselves and with the values found in Ref. [27]). Let us emphasize again that the continuum limits are compatible only when $f_{\pi}^{\text {ov }}$ is extracted from the PP-SS correlator, i.e. when the contribution of zero modes is removed. The necessity of using proper matching conditions as found in our work should, in our opinion, be taken into account for any calculation in a mixed action setup which uses chiral invariant fermions in the quark sector.

In order to further corroborate this conclusion we also tried the naive matching condition, using solely the PP correlator, for a larger volume with $L \approx 2 \mathrm{fm}$ and a pion mass of $m_{\pi} \approx 480 \mathrm{MeV}$, i.e. the ensemble $B_{s}$ of Tab. 1. For this ensemble, we could observe a significant drop of the difference between $f_{\pi}^{\text {ov }}$ and $f_{\pi}^{\text {tm }}$ from about $40 \%$ for the small volume and small pion mass to about only $8 \%$ for the ensemble $B_{s}$. Details of this analysis are presented in Appendix A.

\section{Low energy constants of mixed action chiral perturbation theory}

\subsection{Pseudoscalar meson masses and $\Delta_{M i x}$}

The chiral Lagrangian including $\mathcal{O}\left(a^{2}\right)$ corrections for a mixed action with twisted mass sea and Ginsparg-Wilson valence quarks can be derived from the twisted mass chiral Lagrangian [56, 57, 58, 59, 60]. In this section we will use the expressions of Ref. [61]. They contain the Wilson chiral perturbation theory $(\chi \mathrm{PT})$ low energy constants (LECs) $W_{0}, W_{6,7,8}^{\prime}$ as well as an additional LEC, $W_{M}$, appearing in a mixed action. A dependence on the twist angle $\omega_{0}$, defined by $\operatorname{atan}\left(\omega_{0}\right)=\mu / m_{0}$ is also present.

Considering the power-counting $a^{2} \sim m$ leads to the following leading order (LO) expressions for sea and valence quark mass dependence of the 
light pseudoscalar meson masses [62, 63, 64, 61, 65, 66] (we set $\omega_{0}=\pi / 2$ ):

$$
\begin{aligned}
M_{\mathrm{SS}, \pm}^{2} & =2 B_{0} \mu \\
M_{\mathrm{SS}, 0, \mathrm{conn}}^{2} & =2 B_{0} \mu-\hat{a}^{2} \frac{32}{f^{2}} W_{8}^{\prime}, \\
M_{\mathrm{VV}}^{2} & =2 B_{0} m_{\mathrm{ov}} \\
M_{\mathrm{VS}}^{2} & =B_{0}\left(m_{\mathrm{ov}}+\mu\right)+\hat{a}^{2} \frac{4}{f^{2}} W_{M}-\hat{a}^{2} \frac{8}{f^{2}} W_{8}^{\prime},
\end{aligned}
$$

where the convention for the pion decay constant in the chiral limit $f$ is such that $f_{\pi}$ is $132 \mathrm{MeV}, B_{0}$ is a low energy constant related to the chiral condensate: $B_{0}=-2\langle 0|\bar{u} u| 0\rangle / f^{2}$ [67], $\hat{a}=2 W_{0} a, M_{\mathrm{SS}, \pm}$ is the charged seasea (SS) meson mass, $M_{\mathrm{SS}, 0 \text {,conn }}$ the neutral sea-sea meson mass computed solely from the quark-connected contributions and $M_{\mathrm{VV}}, M_{\mathrm{Vs}}$ are valencevalence (VV) and valence-sea (VS) meson masses, respectively.

Rearranging the above expressions, one can find the relation between the dimensionless combination of LECs $\left(r_{0}^{6} W_{0}^{2}\right)\left(W_{M}-2 W_{8}^{\prime}\right)$ and the SS, VV and VS meson masses:

$$
\left(r_{0}^{6} W_{0}^{2}\right)\left(W_{M}-2 W_{8}^{\prime}\right)=r_{0}^{2}\left(M_{\mathrm{VS}}^{2}-\frac{M_{\mathrm{VV}}^{2}+M_{\mathrm{SS}, \pm}^{2}}{2}\right) \frac{\left(r_{0} f\right)^{2}}{16}\left(\frac{r_{0}}{a}\right)^{2} .
$$

This equation will allow us to determine the combination $\left(r_{0}^{6} W_{0}^{2}\right)\left(W_{M}-2 W_{8}^{\prime}\right)$ from our lattice data for the masses $M_{\mathrm{VV}}, M_{\mathrm{SS}, \pm}$ and $M_{\mathrm{VS}}$.

An alternative parametrization of the VS meson mass [62, 63, 64] is:

$$
M_{\mathrm{VS}}^{2}=B_{0}\left(m_{\mathrm{ov}}+\mu\right)+a^{2} \Delta_{M i x} .
$$

The relation between $\left(W_{M}-2 W_{8}^{\prime}\right)$ and $\Delta_{M i x}$ is the following:

$$
\Delta_{M i x}=\frac{16 W_{0}^{2}\left(W_{M}-2 W_{8}^{\prime}\right)}{f^{2}} .
$$

For our ensembles $B_{\ell}, C_{\ell}, D_{\ell}$ and $B_{h}$, we have computed the pseudoscalar correlation functions for mesons constructed from two MTM quarks (SS, \pm ), two overlap quarks (VV) and a combination of one MTM and one overlap quark (VS). We will call the latter mixed correlators. From each of these correlators, we have extracted pseudoscalar meson masses. The dependence of these masses $\left(M_{\mathrm{SS}, \pm}, M_{\mathrm{VV}}\right.$ and $M_{\mathrm{VS}}$, respectively) on the quark mass is shown in Fig. 10. Since the combination of LECs $\left(r_{0}^{6} W_{0}^{2}\right)\left(W_{M}-2 W_{8}^{\prime}\right)$ and hence also $\Delta_{M i x}$ are determined by the difference between $M_{\mathrm{VS}}^{2}$ and the average of $M_{\mathrm{VV}}^{2}$ and $M_{\mathrm{SS}, \pm}^{2}$, we use the so determined meson masses in the 

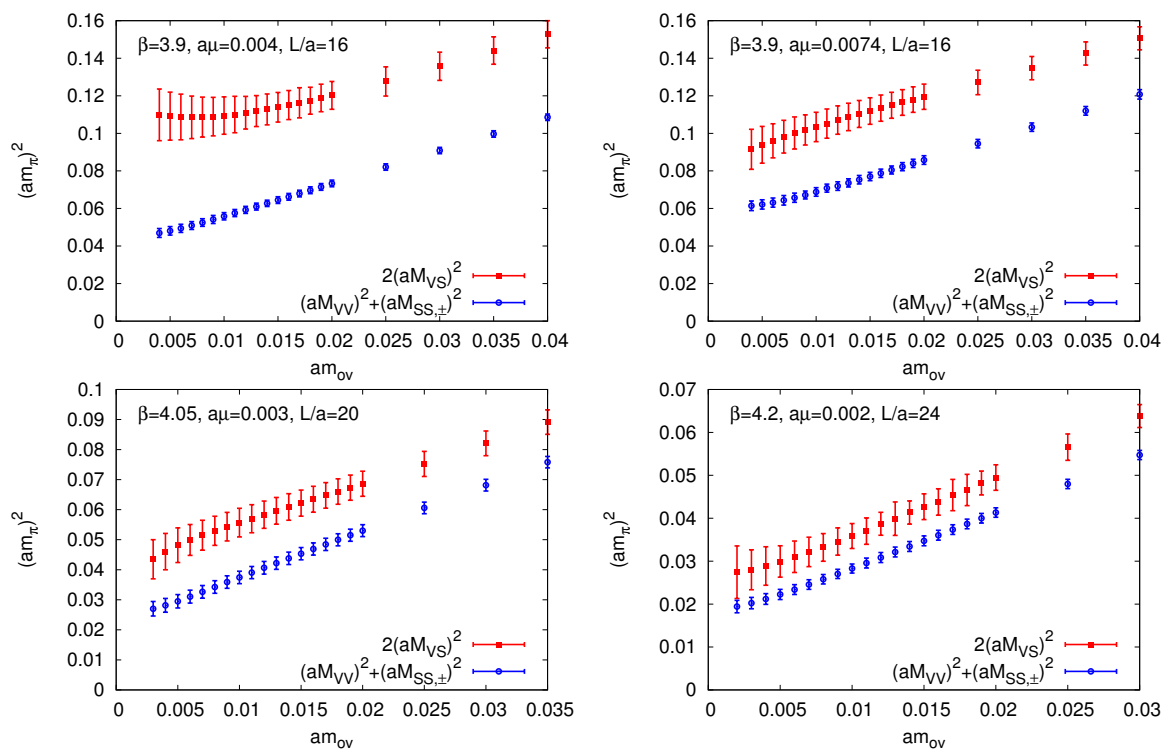

Figure 10: The quark mass dependence of the mixed (VS) pseudoscalar meson mass and the overlap (VV) pseudoscalar meson mass for the ensembles of Tab. 1. The latter has been shifted by the unitary MTM (SS, \pm ) pseudoscalar meson mass, which does not depend on $m_{\mathrm{ov}}$. The quark mass independent vertical distance between $2\left(a M_{\mathrm{VS}}\right)^{2}$ and $\left(a M_{\mathrm{VV}}\right)^{2}+\left(a M_{\mathrm{SS}, \pm}\right)^{2}$ determines the value of $\Delta_{M i x}$, which should in the LO of $\chi \mathrm{PT}$ considered here not depend on the quark mass.

graph. At the considered order of $\chi \mathrm{PT}$, this difference should not depend on the overlap quark mass $m_{\mathrm{ov}}$ and the twisted quark mass $\mu$, i.e. the data for $M_{\mathrm{VS}}^{2}$ and $M_{\mathrm{VV}}^{2}+M_{\mathrm{SS}, \pm}^{2}$ should be parallel as a function of the quark mass.

As the Fig. 10 shows, this is indeed the case for all ensembles we have investigated. In Tab. 6, we also show the extracted values of the combination $\left(r_{0}^{6} W_{0}^{2}\right)\left(W_{M}-2 W_{8}^{\prime}\right)$ and the corresponding values of $\Delta_{M i x}$. The overlap quark mass dependence of these LECs is compatible with a constant behaviour and hence we have used constant fits of the difference $2 M_{\mathrm{VS}}^{2}-\left(M_{\mathrm{VV}}^{2}+M_{\mathrm{SS}, \pm}^{2}\right)$ to extract $\left(r_{0}^{6} W_{0}^{2}\right)\left(W_{M}-2 W_{8}^{\prime}\right)$ for each ensemble, see also Fig. 11. We find that the extracted values are compatible for all ensembles, as they should at his order of $\chi \mathrm{PT}$. In fact, the spread in $\left(r_{0}^{6} W_{0}^{2}\right)\left(W_{M}-2 W_{8}^{\prime}\right)$ (or in $\Delta_{M i x}$ ) between our light sea quark ensembles, labeled with the subscript $\ell$, is very small, suggesting that lattice spacing effects are rather small, at least within the precision we could reach here.

As our final values for $\left(r_{0}^{6} W_{0}^{2}\right)\left(W_{M}-2 W_{8}^{\prime}\right)$ and $\Delta_{M i x}^{1 / 4}$, we take the weighted averages of the values from Tab. 6 (with the inverse statistical error squared 

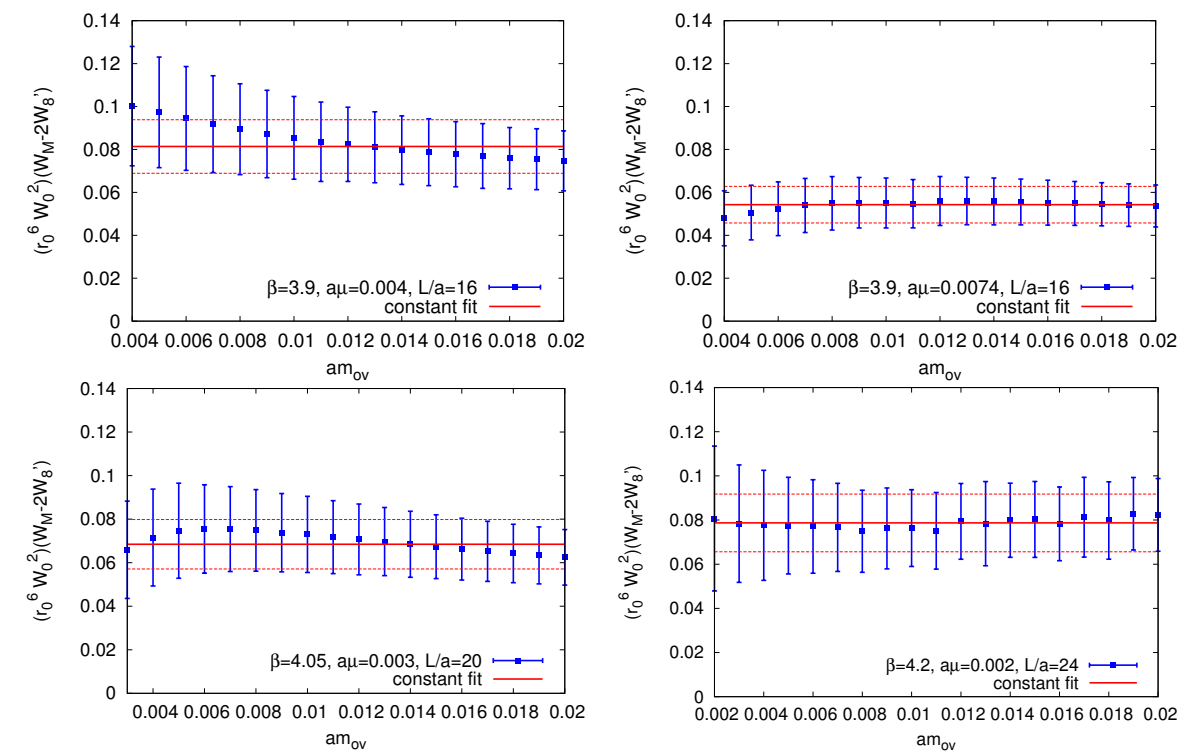

Figure 11: The quark mass dependence of the combination of LECs $\left(r_{0}^{6} W_{0}^{2}\right)\left(W_{M}-2 W_{8}^{\prime}\right)$ for the ensembles of Tab. 1. The vertical scale is the same on all plots, indicating that the values extracted for all ensembles are compatible with each other.

\begin{tabular}{ccccc}
\hline \hline \multirow{2}{*}{ Ensemble } & matching & \multicolumn{2}{c}{$\left(r_{0}^{6} W_{0}^{2}\right)\left(W_{M}-2 W_{8}^{\prime}\right)$} & $\Delta_{M i x}^{1 / 4}$ \\
& $a m_{o v}$ & at matching mass & constant fit & {$[\mathrm{MeV}]$} \\
\hline$B_{\ell}$ & 0.007 & $0.092(23)$ & $0.081(12)$ & $997(37)(21)$ \\
$C_{\ell}$ & 0.005 & $0.075(22)$ & $0.068(11)$ & $951(38)(21)$ \\
$D_{\ell}$ & 0.002 & $0.081(33)$ & $0.079(13)$ & $968(40)(21)$ \\
$B_{h}$ & 0.015 & $0.056(11)$ & $0.054(9)$ & $901(38)(20)$ \\
\hline \hline
\end{tabular}

Table 6: The extracted values of the combination of LECs $\left(r_{0}^{6} W_{0}^{2}\right)\left(W_{M}-2 W_{8}^{\prime}\right)$. We show the values at the matching point $M_{\mathrm{VV}}=M_{\mathrm{SS}, \pm}$ (the matching quark mass is given in the column $a m_{\mathrm{ov}}$ ) and from the constant fit to $2\left(a M_{\mathrm{VS}}\right)^{2}-\left(a M_{\mathrm{VV}}\right)^{2}+\left(a M_{\mathrm{SS}, \pm}\right)^{2}$ for the whole quark mass range shown in Fig. 11, The last column is $\Delta_{M i x}^{1 / 4}$ in $\mathrm{MeV}$, for which the first error is statistical and the second one the systematic error of conversion to $\mathrm{MeV}$, coming from uncertainties in the values of lattice spacings. 
as the weight). This yields:

$$
\begin{gathered}
\left(r_{0}^{6} W_{0}^{2}\right)\left(W_{M}-2 W_{8}^{\prime}\right)=0.067(6)(14), \\
\Delta_{M i x}=[951(21)(50) \mathrm{MeV}]^{4},
\end{gathered}
$$

where the first error is statistical (error of the weighted average) and the second one is systematic, which we take as one half of the spread between the values for the ensembles $B_{\ell}$ and $B_{h}$. This implies an approx. $140 \mathrm{MeV}$ splitting between the VS and VV meson masses at the matching mass, for ensemble $B_{\ell}$. Note that our result for $W_{M}-2 W_{8}^{\prime}$ is compatible with the constraint $W_{M}-2 W_{8}^{\prime} \geq 0$ 6, found in Ref. [65].

The value of $\Delta_{M i x}$ that we have determined is comparable to, but somewhat larger in comparison with previous studies with different mixed action setups: $\Delta_{M i x}=[706(4) \mathrm{MeV}]^{4}\left[68\right.$ and $\left[678(13) \mathrm{MeV}^{4}\right.$ (with a finer lattice spacing) 21 for domain wall fermions on a staggered sea, $[769(77) \mathrm{MeV}]^{4}$ and $\left[861(90) \mathrm{MeV}^{4}\right.$ (for a heavier pion mass) [17] for overlap fermions on a clover sea and $-\left[427(338) \mathrm{MeV}^{4}[16]\right.$ or $[416(27) \mathrm{MeV}]^{4}$ [69] for overlap fermions on a domain wall sea 7 .

\subsection{Unitarity violations in the scalar correlator}

\subsubsection{Theoretical predictions}

Mixed actions, even when related to each other by suitable matching conditions, violate unitarity at any non-vanishing value of the lattice spacing. Unitarity is then only restored in the continuum limit. So far, we have looked at unitarity violations in the pion decay constant at the matching mass and in mixed correlators. Here, we investigate yet another way of studying unitarity violations, through a quantity which is known to depend strongly on them and, in principle, allows to quantify their size - the flavour non-singlet scalar correlator. The reason is that within $\chi \mathrm{PT}$, unitarity violations appear as unphysical double poles in the propagator, affecting strongly the flavour non-singlet scalar correlator even at the matching point, i.e. if the valencevalence (VV) pseudoscalar meson mass is matched to the sea-sea (SS) meson mass [70, 171, 172, 73]. In fact, the scalar correlator can turn negative in a mixed action setup which is clearly an unphysical behaviour.

\footnotetext{
${ }^{6}$ In Ref. 65, the constraint takes the form $2 W_{M}-W_{8}^{\prime} \geq 0$. However, $W_{M}$ in our notation receives a factor of 4 compared to the one defined in Ref. 65.

${ }^{7}$ The former value has been obtained using a different (indirect) method - by examining a meson state that wraps around the time boundary, instead of a direct extraction from mixed correlators.
} 
The flavour non-singlet scalar correlator can be approximated by: [61]

$$
\begin{aligned}
C_{\mathrm{sca}}^{\mathrm{VV}}(t)= & -2\left(M_{\mathrm{SS}, \pm}^{2}-M_{\mathrm{VV}}^{2}+\hat{a}^{2} \frac{16}{f^{2}} W_{8}^{\prime}\right) \cdot B_{\mathrm{DP}}\left(T, L, t, M_{\mathrm{VV}}, M_{\mathrm{VV}}\right) \\
& -2 B_{\mathrm{SP}}\left(T, L, t, M_{\mathrm{VV}}, M_{\mathrm{VV}}\right)+2 B_{\mathrm{SP}}\left(T, L, t, M_{\mathrm{VS}}, M_{\mathrm{VS}}\right) \\
& +A e^{-m_{a_{0}} t}+\text { excited states }
\end{aligned}
$$

where $B_{\mathrm{SP}}$ and $B_{\mathrm{DP}}$ are the single and double pole bubble functions including finite volume effects [61] and the last terms describe contributions from scalar mesons (the lightest of which is $a_{0}$ ). The label $\mathrm{VV}$ in $C_{\mathrm{sca}}^{\mathrm{VV}}(t)$ reminds that overlap valence quarks are used in the scalar correlator.

Assuming matching of pseudoscalar meson masses $M_{\mathrm{VV}}=M_{\mathrm{SS}, \pm}$, in the limit $T \rightarrow \infty$, and at large Euclidean times $t$, the bubble contributions can be simplified to yield:

$$
C_{\mathrm{sca}}^{\mathrm{VV}}(t) \rightarrow \frac{B_{0}^{2}}{2 L^{3}}\left[\frac{e^{-2 M_{\mathrm{VS}} t}}{M_{\mathrm{VS}}^{2}}-\frac{e^{-2 M_{\mathrm{Vv}} t}}{M_{\mathrm{VV}}^{4}}\left(M_{\mathrm{VV}}^{2}+\hat{a}^{2} \frac{16}{f^{2}} W_{8}^{\prime}\left(1+M_{\mathrm{VV}} t\right)\right)\right] .
$$

Identifying the LEC $\gamma_{\mathrm{SS}}$ in Ref. [73] with $\gamma_{\mathrm{SS}}=\frac{16}{f^{2}} W_{0} W_{8}^{\prime}$, one recovers, in the large $t$ limit, the corresponding expression of Eq. (23) of Ref. [73].

\subsubsection{Lattice results}

Due to the negative coefficients appearing in (26) it can be that the SS correlator becomes negative at large Euclidean times thus indicating a lack of unitarity. However, in order to isolate the different contributions to the violations of unitarity, it is useful to disentangle the effect of zero modes, which can have a potentially very large contribution to the SS correlator. This contribution is actually equal to the zero modes contribution to the $\mathrm{PP}$ correlator. As previously discussed, this is behind the choice of the improved matching condition, which allowed us to extract the pseudoscalar meson mass and decay constant by computing the PP-SS correlator, where the effect of zero modes is exactly canceled. In Fig. 12, we show the scalar correlator for ensembles $B_{\ell}$ and $D_{\ell}$. We have computed the index of the overlap Dirac operator for each configuration and we have calculated the SS correlator for all configurations with a given index. For large Euclidean times (i.e. in the region where Eq. (26) should be applicable), the SS correlator has a tendency to become more and more negative for configurations with an increasing number of zero modes, i.e. in higher topological charge sectors. When the correlator is evaluated on all configurations, the SS correlator is clearly negative, which signals unitarity violations. 

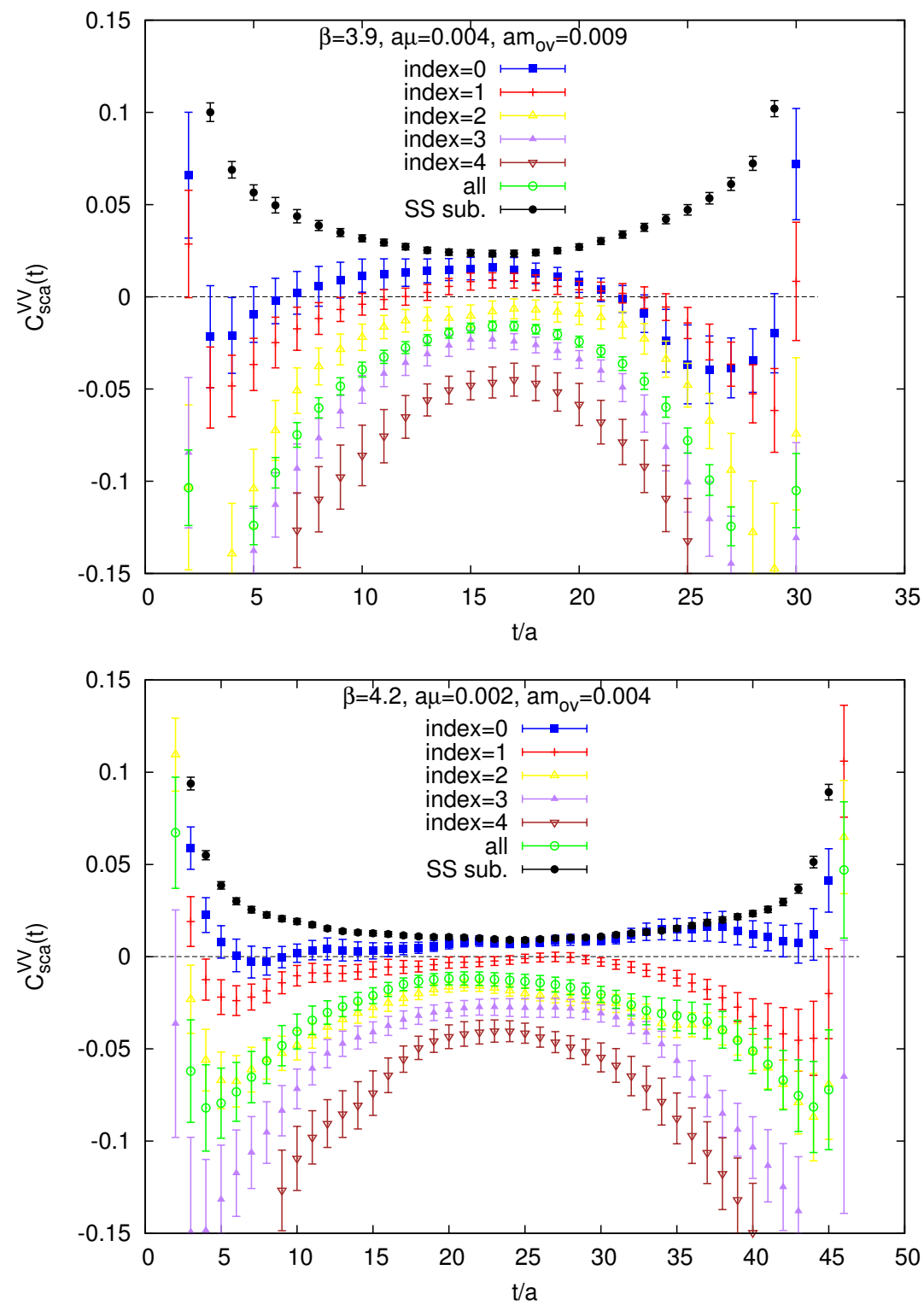

Figure 12: The scalar correlator $C_{\mathrm{sca}}^{\mathrm{VV}}(t)$ in fixed topological charge sectors (index $0,1,2,3,4)$ and from summing over all topological charge sectors (all). In addition, we show the results for the scalar correlator with explicitly subtracted zero modes (SS sub.). Upper: ensemble $B_{l}, \beta=3.9, L / a=16$, $a m_{s}=0.004, a m_{\mathrm{ov}}=0.009$. Lower: ensemble $D_{l}, \beta=4.2, L / a=24$, $a m_{s}=0.002, a m_{\mathrm{ov}}=0.004$. 


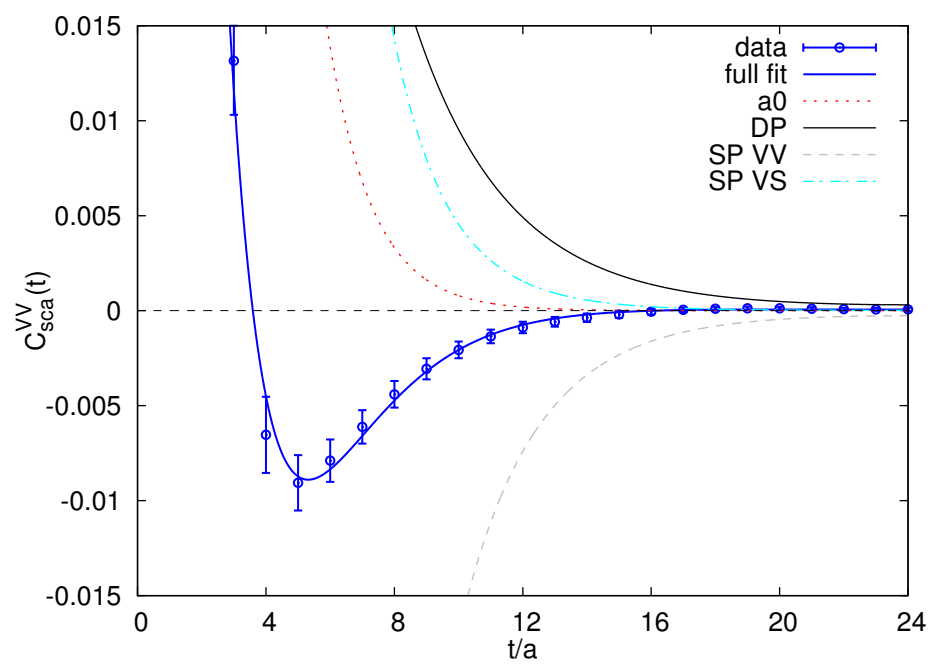

Figure 13: Fits of Eq. (25), using ensemble $B_{s}$, for which the zero modes effects are very much suppressed. Blue solid line is the full fit of Eq. (25), which is a sum of 4 contributions: double pole (DP) - black solid line, single pole containing $M_{V V}(\mathrm{SP} V \mathrm{~V})$ - gray dashed line, single pole containing $M_{V S}$ (SP VS) - cyan dash-dotted line, scalar $a_{0}$ meson (a0) - red dotted line.

In order to determine the low energy constant $W_{8}^{\prime}$ appearing in Eq. (25), it is much safer to use larger volumes and quark masses, i.e. a situation when the contribution of zero modes is highly suppressed. Among our ensembles, $B_{s}$ has the desired properties and will be used to extract $W_{8}^{\prime}$.

Another possibility is to restrict oneself to topologically trivial configurations. However, it is clear that the number of such configurations is rather small for each ensemble (between 10 and $20 \%$ of all configurations) and hence the signal is of poor quality, as can also be inferred from Fig. 12 directly. Moreover, taking only topologically trivial configurations means that the results of the ensemble average will differ from the full result (i.e. from all topological sectors) by power-like finite volume effects [35, 74]. Therefore, such an analysis will be used only as a cross-check and as an estimate of a systematic error related to our computation using ensemble $B_{s}$.

Yet another way to proceed is to explicitly subtract the contribution of zero modes at the level of propagators. This is a potentially dangerous procedure with hard-to-control systematic effects. The SS correlator with explicitly subtracted zero modes (which we call $S S$ subtr.) is shown in Fig. 12. However, given the fact that the zero mode subtraction is a rather doubtful procedure, we will quote the value obtained with this method, but we will 

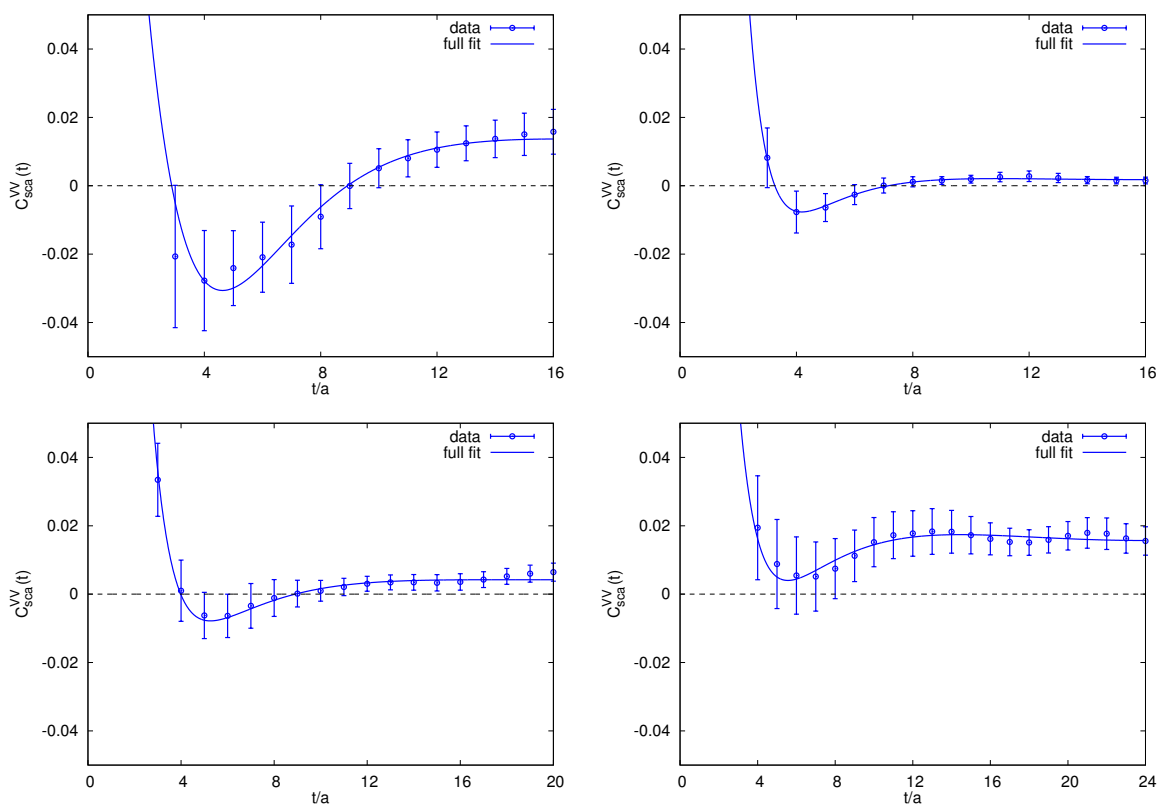

Figure 14: Fits of Eq. (25) to the SS correlators for the small volume ensembles $B_{\ell}$ (upper left), $B_{h}$ (upper right), $C_{\ell}$ (lower left), $D_{\ell}$ (lower right).

not take it into account in our analysis leading to the final value of $W_{8}^{\prime}$.

We now describe our strategy to extract $W_{8}^{\prime}$ from ensemble $B_{s}$, using ensembles $B_{\ell}, B_{h}, C_{\ell}$ and $D_{\ell}$ to estimate the systematic error. We fit Eq. (25), which has 3 fitting parameters: the LEC of interest $-W_{8}^{\prime}$, the amplitude of $a_{0}$ scalar meson contribution and its mass $m_{a_{0}}$ (however, we do not attempt to extract the $a_{0}$ contribution quantitatively). In addition, we add a fourth fitting parameter - the overall normalization of the correlator $N$, into which we absorb unknown renormalization factors $Z_{S}$. We work with symmetrized correlators and take into account the finite extent of the system by adding in Eq. (25) the terms with $t \rightarrow T-t$.

The results of our fits for ensemble $B_{s}$ are shown in Fig. 13, The dimensionless combination of LECs $r_{0}^{6} W_{0}^{2} W_{8}^{\prime}$ determined from our best fit is $-0.00642(15)$, where the error is statistical only. We now want to determine the systematic error, related to: residual discretization effects, finite volume effects, choice of the Euclidean time fit range and finite precision of the input parameters of our fits: $a M_{\mathrm{VV}}=0.1918(12)$ at the matching mass $a m_{\mathrm{ov}}=0.017$ (see Appendix 胃), $a M_{\mathrm{SS}, \pm}=0.19403(50) \sqrt{8}[44], \Delta_{M i x}$ (taken

\footnotetext{
${ }^{8}$ Note that $a M_{\mathrm{VV}}$ is not exactly equal to $a M_{\mathrm{SS}, \pm}$, which we take into account by including the difference $M_{\mathrm{SS}, \pm}^{2}-M_{\mathrm{VV}}^{2}$ in the fits of Eq. (25).
} 


\begin{tabular}{lcc}
\hline \hline central value & -0.0064 & $B_{s}$ \\
\hline fit range & 0.0015 & $B_{s}$ \\
$\Delta_{M i x}$ & 0.0004 & $B_{s}$ \\
$a M_{V V}$ & 0.0002 & $B_{s}$ \\
$a M_{S S}$ & 0.0001 & $B_{s}$ \\
including/excluding $M_{\mathrm{SS}, \pm}^{2}-M_{\mathrm{VV}}^{2}$ & 0.0003 & $B_{s}$ \\
discretization effects & 0.0008 & $B_{\ell}, C_{\ell}, D_{\ell}$ \\
finite volume & 0.0009 & $B_{s}, B_{h}$ \\
sea quark mass & 0.0012 & $B_{\ell}, B_{h}$ \\
$r_{0} / a, r_{0} f$ & 0.0006 & $\operatorname{Ref.~[39]}$ \\
\hline total systematic & 0.0024 & $B_{s}, B_{h}, B_{\ell}, C_{\ell}, D_{\ell}$ \\
statistical & 0.0002 & $B_{s}$ \\
\hline
\end{tabular}

Table 7: Error budget for our estimates of the combination of LECs $r_{0}^{6} W_{0}^{2} W_{8}^{\prime}$. The last column shows which ensembles were used to determine the given type of systematic error (or central value). The total systematic error comes from combining the individual ones in quadrature.

from the previous subsection), $r_{0} / a=5.25(2), r_{0} f=0.259(11)$ [39].

We address the errors related to discretization effects, finite volume effects and sea quark mass using our small-volume ensembles at 3 lattice spacings: $B_{\ell}, C_{\ell}, D_{\ell}$ (at a pion mass of about $300 \mathrm{MeV}$ ) and $B_{h}$ (approximately 450 $\mathrm{MeV})$. We illustrate fits for these ensembles in Fig. 14. The remaining systematic errors were estimated using ensemble $B_{s}$ only and varying the respective quantities, in particular the fitting range. We have also checked the stability of our results with respect to including or excluding the 2 fitting parameters related to the scalar meson $\left(a_{0}, A\right)$. This gives fully compatible results (within the systematic error from the choice of the fitting range), however we are then limited to larger values of $t / a$. The summary of all systematic errors is presented in Tab. 7 .

The final value that we quote is:

$$
r_{0}^{6} W_{0}^{2} W_{8}^{\prime}=-0.0064(2)(24)
$$

where the first error is statistical and the second systematic. This value can be compared to the recent determination (with twisted mass fermions) [75] from the connected contribution to the neutral pion correlator (Eq. (19)), which gives $r_{0}^{6} W_{0}^{2} W_{8}^{\prime}=-0.0106(11)$ (ensemble $B_{\ell}$ at a larger volume with $L / a=24$ instead of 16 ) or $r_{0}^{6} W_{0}^{2} W_{8}^{\prime}=-0.0096(22)$ (ensemble $B_{\ell}$ ). We also give here as a cross-check the value obtained from explicit subtraction of 


\begin{tabular}{cc|cc}
\hline \hline$\left(r_{0}^{6} W_{0}^{2}\right)\left(W_{M}-2 W_{8}^{\prime}\right)$ & $0.067(6)(14)$ & $\Delta_{M i x}$ & {$[951(21)(50) \mathrm{MeV}]^{4}$} \\
$\left(r_{0}^{6} W_{0}^{2}\right) W_{8}^{\prime}$ & $-0.0064(2)(24)$ & $w_{8}^{\prime}$ & $-[529(4)(51) \mathrm{MeV}]^{4}$ \\
$\left(r_{0}^{6} W_{0}^{2}\right) W_{M}$ & $0.054(6)(15)$ & $w_{M}$ & {$[901(21)(62) \mathrm{MeV}]^{4}$} \\
\hline \hline
\end{tabular}

Table 8: The values for different combinations of LECs determined in this work. The first error is statistical and the second one systematic.

zero modes: $r_{0}^{6} W_{0}^{2} W_{8}^{\prime}=-0.0127(8)$ (where the error is purely statistical). Although this value has the right order of magnitude, we emphasize again that the validity of the explicit zero modes subtraction procedure is doubtful and hence this result should be interpreted with caution. We also note that our finding for $W_{8}^{\prime}$ is compatible with the constraint $W_{8}^{\prime} \leq 0$ found in Refs. [66, 76, 77, 78].

Our results for the combination of LECs $r_{0}^{6} W_{0}^{2} W_{8}^{\prime}$ and $r_{0}^{6} W_{0}^{2}\left(W_{M}-2 W_{8}^{\prime}\right)$ can be combined to isolate:

$$
r_{0}^{6} W_{0}^{2} W_{M}=0.054(6)(15),
$$

where the meaning of the two errors is as above.

We also define quantities analogous to $\Delta_{M i x}$ :

$$
\begin{gathered}
w_{8}^{\prime}=\frac{16 W_{0}^{2} W_{8}^{\prime}}{f^{2}}, \\
w_{M}=\frac{16 W_{0}^{2} W_{M}}{f^{2}} .
\end{gathered}
$$

With such definitions, the following relation holds: $\Delta_{M i x}=w_{M}-2 w_{8}^{\prime}$. Our results imply the values: $w_{M}=[901(21)(62) \mathrm{MeV}]^{4}$ and $w_{8}^{\prime}=-[529(4)(51) \mathrm{MeV}]^{4}$. The latter corresponds to approximately $125 \mathrm{MeV}$ splitting between the charged and neutral (connected 9 ) pion masses, for ensemble $B_{\ell}$. For convenience, we summarize all our results for mixed action $\chi$ PT LECs in Tab. 8 ,

\section{$6 \quad$ Light baryon masses}

In order to investigate the effects of the mixed action setup in other observables, we have computed the nucleon and $\Delta$ baryon masses in the mixed

\footnotetext{
${ }^{9}$ If one considers the full (connected and disconnected contributions) neutral pion mass $M_{\mathrm{SS}, 0}$, the splitting $M_{\mathrm{SS}, 0}-M_{\mathrm{SS}, \pm}$ is governed by a combination of LECs $W_{6}^{\prime}$ and $W_{8}^{\prime}$ (see e.g. Ref. 60]). The LEC $W_{6}^{\prime}$ is not directly accessible from the overlap scalar correlator.
} 
action and the unitary setup. Given the numerical cost of the inversion of the overlap operator, we performed the analysis on the following subset of gauge ensembles generated on only small lattice sizes up to $24^{3} \cdot 48, B_{\ell}(\beta=3.9)$, $C_{\ell}(\beta=4.05)$ and $D_{\ell}(\beta=4.20)$ that correspond to a fixed spatial extent of $\approx 1.3 \mathrm{fm}$ and a fixed pseudo scalar mass of $\sim 300 \mathrm{MeV}$. Having three values of the lattice spacing available, allows us to study the continuum limit for the nucleon $(\mathrm{N})$ and $\Delta$ masses in both setups. The strategy followed to extract the baryon masses in this section follows closely the work on the light [79] and strange baryon spectrum [53] by ETMC. For consistency, we recall here the basic ingredients of the computation, the reader interested in more details is referred to the two aforementioned references.

The masses of the nucleon and of $\Delta$ baryons are computed through the two-point correlators, using the following interpolating fields:

$$
\begin{aligned}
& J^{N}=\epsilon^{a b c}\left(u^{a, T} C \gamma_{5} d^{b}\right) u^{c}, \\
& J_{\mu}^{\Delta}=\epsilon^{a b c}\left(u^{a, T} C \gamma_{\mu} u^{b}\right) u^{c},
\end{aligned}
$$

where $C$ is the charge conjugation matrix. The quark fields $u$ and $d$ refer to the two degenerate flavours of quarks considered in this work 10 While the operator $J^{N}$ carries spin $1 / 2$ and couples only to states which have the quantum numbers of the nucleon, the operator $J_{\mu}^{\Delta}$ couples both to spin $1 / 2$ and spin $3 / 2$ states. Note, however, that in practical lattice computations the spin $3 / 2$ dominates the correlator at large time [79] thus allowing for a clean extraction of the $\Delta$ baryon. As in nature, the spin $1 / 2$ partner is much heavier than the spin $3 / 2$ state.

In order to improve the overlap between the ground state and the interpolating fields, we employ source and sink smearing of the quark fields. We use Gaussian smearing [80, 81] of the quarks field and APE smearing 82 of the gauge links entering in the Gaussian smearing procedure. We use the smearing parameters obtained in our previous works on baryon spectroscopy [79, 53. Note that we use the same smearing parameters both in the twisted mass and overlap fermion cases.

In order to investigate the consequences of the various matching conditions discussed in Sec. 4, we use several valence overlap quark masses. The baryon masses are extracted from the asymptotic Euclidean time behaviour of the correlators:

\footnotetext{
${ }^{10}$ In the unitary case, the $u$ and $d$ quarks refer to the fields in the so-called physical basis.
} 


$$
C^{N, \Delta}(t)=\sum_{\vec{x}} \frac{1+\gamma_{0}}{2}\left\langle J^{N, \Delta}(\vec{x}, t) \bar{J}^{N, \Delta}(0)\right\rangle-\frac{1-\gamma_{0}}{2}\left\langle J^{N, \Delta}(\vec{x}, t-T) \bar{J}^{N, \Delta}(0)\right\rangle,
$$

The statistical errors are estimated using 1000 bootstrap samples. Concerning the topological finite size effects, isolating the zero mode contribution after the fermionic integration, the leading behaviour of Eq. (31) is:

$$
C(t) \sim\left(a m_{\mathrm{ov}}\right)^{-3}
$$

as discussed in Ref. 83.

We use the effective masses defined by:

$$
m_{\mathrm{eff}}^{N, \Delta}(t)=-\log \frac{C^{N, \Delta}(t)}{C^{N, \Delta}(t-1)}=a m_{N, \Delta}+\mathcal{O}\left(e^{-\delta t}\right)
$$

where $\delta$ is the mass difference between the ground state and the first excited state. In Fig. 15, we show examples of effective masses of the nucleon and $\Delta$ in the mixed action setup. The results are obtained at the coarsest and the finest lattice spacing used in this work, corresponding to the gauge ensembles $B_{\ell}$ (left) and $D_{\ell}$ (right). The valence quark masses are fixed to the (improved) matching overlap quark masses summarized in Tab. 5 We fit the effective masses to a constant in the Euclidean time region where $m_{\mathrm{eff}}^{N, \Delta}(t)$ become time independent. The corresponding fits are shown in Fig. 15] where the fit result is represented by horizontal black lines and their statistical errors by black dotted lines. The effective mass plots in Fig. 15] show that we can identify a plateau region, allowing us a good determination of the nucleon and the $\Delta$ masses.

Results for the nucleon and $\Delta$ masses in the unitary setup and in the mixed action setup (for $a m_{\mathrm{ov}}$ set to the matching mass) are reported in Tab. 9. We also show our results obtained for several valence quark masses in Fig. 16 for ensemble $B_{\ell}$ (left) and for $D_{\ell}$ (right) - each plot shows the overlap quark mass dependence of the nucleon mass (black dots) and the $\Delta$ mass (blue triangles). The matching mass is indicated by a vertical dotted line, while the results obtained in the unitary setup are indicated by a yellow band with a width corresponding to the statistical errors. Note that for very low valence quark masses, below the matching quark mass, a plateau region in the effective mass can hardly be found. This explains why statistical errors become larger for very small overlap quark masses. The uncertainty to extract reliably effective masses in this region is also responsible for the somewhat irregular behaviour in Fig. 16. As can be seen from the very good 

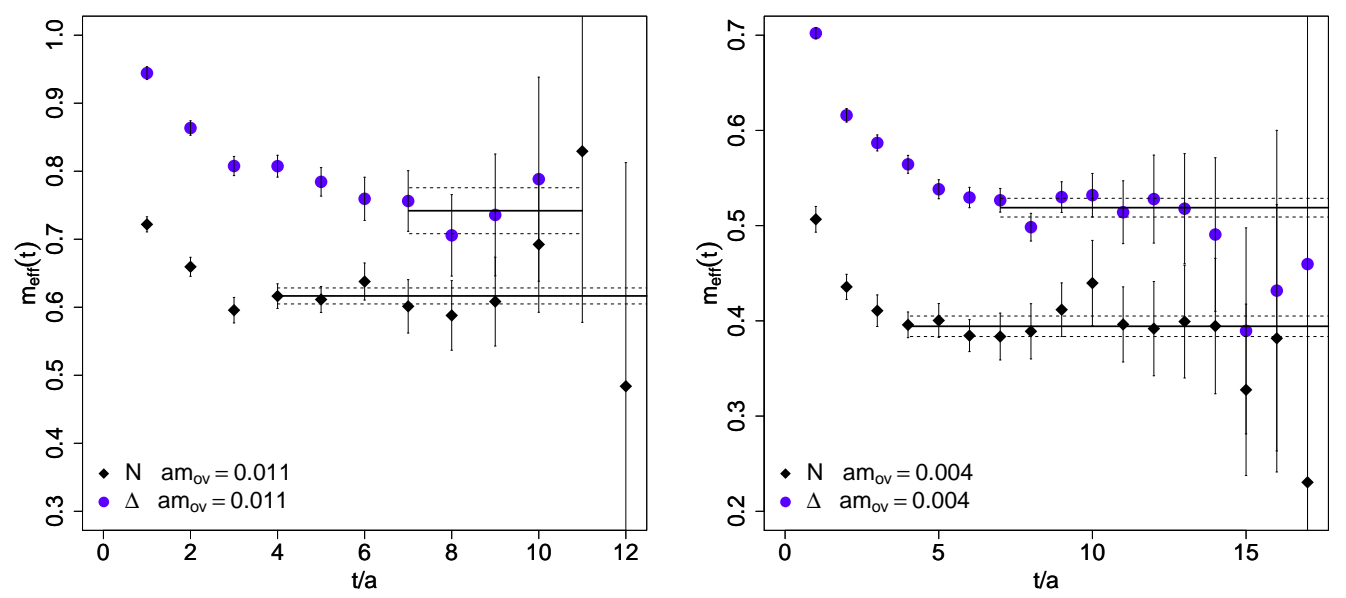

Figure 15: Nucleon and $\Delta$ effective masses in the mixed action setup. The valence quark mass is fixed to the (improved) matching overlap quark mass, see Tab. 5 for their values. In the left plot we show the results obtained for the ensemble $B_{\ell}(\beta=3.9)$ and in the right for $D_{\ell}(\beta=4.2)$.

\begin{tabular}{ccccccc}
\hline Ensemble & $N^{\mathrm{TM}}$ & $N^{\mathrm{ov}}$ & $a m_{N}^{\mathrm{TM}}$ & $a m_{N}^{\mathrm{ov}}$ & $a m_{\Delta}^{\mathrm{TM}}$ & $a m_{\Delta}^{\mathrm{ov}}$ \\
\hline \hline$B_{\ell}$ & 636 & 426 & $0.594(13)$ & $0.617(12)$ & $0.740(24)$ & $0.741(34)$ \\
$C_{l}$ & 286 & 142 & $0.497(11)$ & $0.522(27)$ & $0.627(13)$ & $0.641(15)$ \\
$D_{\ell}$ & 189 & 371 & $0.407(14)$ & $0.394(11)$ & $0.503(13)$ & $0.519(10)$ \\
\hline \hline
\end{tabular}

Table 9: Summary of our results for for the nucleon masses $a m_{N}$ and $\Delta$ masses $a m_{\Delta}$. The superscript "TM" denotes the unitary setup, while "ov" denotes the mixed action setup. We also give the number of gauge field configurations $N$ used for the measurements on each ensemble.

agreement of the baryon masses between the unitary and the mixed action setups, even in a regime where effects of chiral zero modes of the overlap operator are sizable in the pion sector, in the nucleon or $\Delta$ mass case, the effects are negligible for our definition of the (improved) matching mass.

Finally, we show in Fig. 17 the continuum limit scaling plot of the two baryon masses with three lattice spacings, in the unitary and mixed action case. All three ensembles have pion masses of $\approx 300 \mathrm{MeV}$ and the same volume of $\approx 1.3 \mathrm{fm}$, so no interpolation in volume or quark masses is needed within our statistical accuracy to compare our results at different lattice spacings. For all ensembles, the results in the mixed action case and in the 

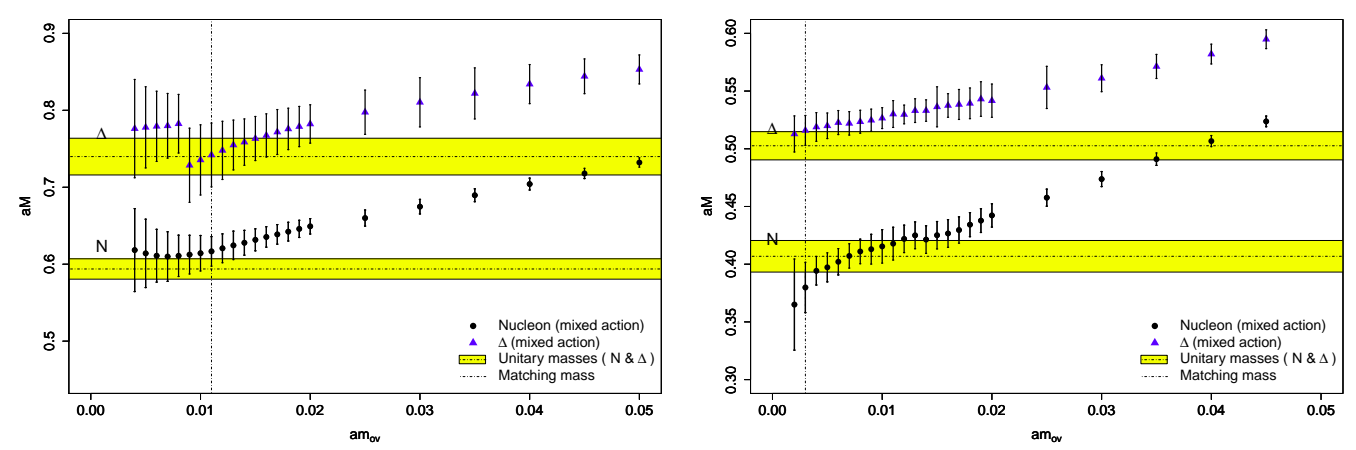

Figure 16: Nucleon and Delta masses vs. $a m_{\mathrm{ov}}$. The vertical dotted line indicates the matching mass. The left plot shows results for ensemble $B_{\ell}$ $(\beta=3.9)$ and the right one for $D_{\ell}(\beta=4.2)$.

unitary case are compatible within errors in the continuum limit. Furthermore, in the two setups, the results for the nucleon and for the $\Delta$ mass show only a small dependence on the lattice spacings. We perform a linear fit in $a^{2}$ represented by dotted lines (red in the unitary case and black in the mixed action one). The continuum extrapolated values are represented slightly shifted for better readability.

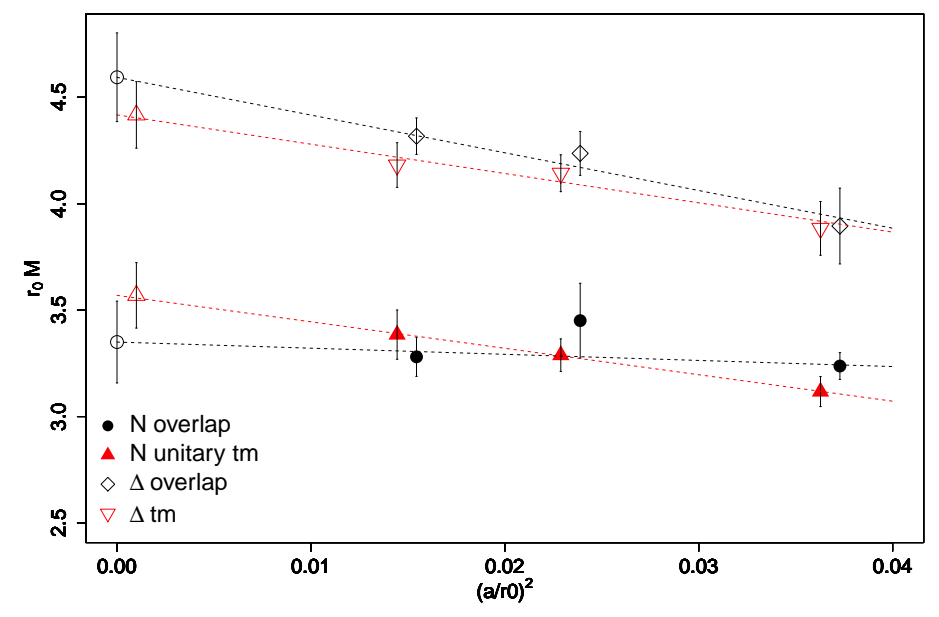

Figure 17: The continuum limit scaling of the nucleon and $\Delta$ masses. 


\section{$7 \quad$ Summary and prospects}

In this paper, we have studied a particular example of a mixed action setup, namely valence overlap fermions on maximally twisted mass sea fermions. By adding a fourth and finer value of the lattice spacing than available to us earlier [27], we were able to perform a continuum limit scaling test for the pion decay constant and to compare the unitary and mixed action theories. We found a continuum limit value of the pion decay decay constant that is in full agreement between the two theories and confirmed thus the conclusion of Ref. 27] that with an improved matching condition the dangerous effects arising from the mismatch of the chiral zero modes - between the chiral invariant overlap Dirac operator in the valence sector and the twisted mass operator in the sea - can be avoided

Applying the same strategy for other observables for which we took the nucleon and $\Delta$ masses, we found that also these quantities are not affected by the presence of the zero modes, once the improved matching condition is applied. This supports the interpretation reached in Ref. [27] that the improved matching condition leads to a proper matching of the sea and valence theories. In general, in the context of mixed actions, we nevertheless advocate to check the possible effects on physical observables of the mismatch of zero modes between valence and sea sectors, introduced by the use of a valence overlap Dirac operator.

We also performed a continuum limit investigation of the locality properties of the overlap Dirac operator. While at any non-zero value of the lattice spacing the overlap Dirac operator is exponentially localized, our study, employing four values of the lattice spacing, strongly suggests that a point-like localization is indeed recovered in the continuum limit. One essential element in our investigation was to compare the exponential decay rate of the norm of the overlap Dirac operator with hadronic scales, for which we took the pion and nucleon masses. We could demonstrate that for all values of the lattice spacing, the ratio of the hadronic masses over the decay rate is smaller than one and assumes a value compatible with zero in the continuum limit. Thus, the exponential locality of the overlap Dirac operator is not expected to distort the evaluation of these hadronic observables. We think that such kind of tests should be performed on hadronic physical quantities one is interested in, in order to monitor the possible distortions of the results by a finite decay rate of the overlap Dirac operator.

Finally, we compared our numerical computations of meson masses with predictions of mixed action $\chi \mathrm{PT}$ and extracted in this way some of the LECs which parametrize the mixed action chiral effective Lagrangian. We also provided evidence that there are indeed unitarity violations in the mixed 
action setup and we quantified these effects by computing the $\Delta_{M i x}$ and $W_{8}^{\prime}$ parameters.

In summary, we believe that the results of Ref. [27, in combination with the investigations performed in this work, can serve as a basis for future mixed action computations employing chiral invariant fermions in the valence sector: we advocate to use the improved matching condition proposed in Ref. [27]; we suggest to test whether the localization rate of the overlap Dirac operator is sufficiently larger than the hadronic scale of interest; we further point to the possibility to use mixed action chiral perturbation theory to look at effects of a mixed action setup, allowing in particular to isolate the effects of unitarity violations.

Acknowledgments We thank the European Twisted Mass Collaboration for generating ensembles of gauge field configurations that we have used for this work and for a very enjoyable collaboration. We are grateful to O. Bär for useful comments and suggestions. Sec. 3 of this work originated from a DESY summer student project carried out by O. Haas, E. Panzer, S. Tordjman. The computer time for this project was made available to us by the Leibniz Rechenzentrum in Munich, Poznań Supercomputing and Networking Center in Poznań, GENCI-CINES grant 2010-052271 and CCIN2P3 in Lyon. We thank these computer centers and their staff for all technical advice and help. K.C. has been supported by Foundation for Polish Science fellowship "Kolumb" and Ministry of Science and Higher Education grant nr. N N202 237437. This work has been supported in part by the DFG Sonderforschungsbereich/Transregio SFB/TR9. G.H. acknowledges the support from the Spanish Ministry for Education and Science project FPA2009-09017, the Consolider-Ingenio 2010 Programme CPAN (CSD2007-00042), the Comunidad Autónoma de Madrid (HEPHACOS P-ESP-00346 and HEPHACOS S2009/ESP-1473) and the European project STRONGnet (PITN-GA-2009238353).

\section{A Results for ensemble $B_{s}$}

One of the main conclusions of Ref. [27] were the values of the relevant parameters - physical volume and pion mass - for which the role of the zero modes is strongly suppressed. With such parameters, the simulation results are safe against the contribution of zero modes, i.e. a continuum limit scaling test of e.g. the pion decay constant, computed with MTM and overlap fermions would lead to a consistent behaviour (same continuum limits), even when both $f_{\pi}^{\mathrm{tm}}$ and $f_{\pi}^{\text {ov }}$ are computed from the PP correlator. 

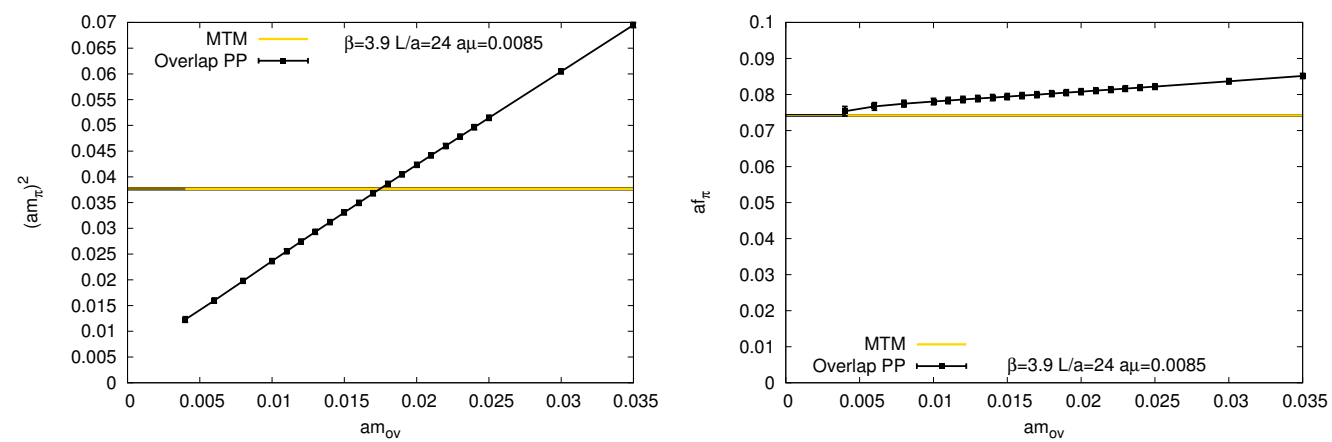

Figure 18: (left) Matching the overlap and MTM pion mass. (right) The dependence of the overlap pion decay constant on the overlap quark mass $a m_{o v}$. The matching mass is around $a m_{\mathrm{ov}}=0.017$. Ensemble $B_{s}(\beta=3.9$, $L / a=24, a \mu=0.0085)$.

For this work, we have decided to test this expectation explicitly, employing an ensemble with large enough volume and pion mass. We have not performed a full continuum limit scaling test, since this is still very expensive computationally. Instead, we compare the values of $f_{\pi}^{\text {tm }}$ and $f_{\pi}^{\text {ov }}$ at the matching mass, expecting only a small difference that can be attributed to $\mathcal{O}\left(a^{2}\right)$ cut-off effects. Our ensemble, labeled $B_{s}$ has the following parameters: $\beta=3.9, L / a=24, a \mu=0.0085$, which corresponds to $L \approx 2 \mathrm{fm}, m_{\pi} \approx 480$ $\mathrm{MeV}$ in infinite volume and $m_{\pi} L \approx 4.7$. The latter is well above $m_{\pi} L=4$, the minimal product of the pion mass and spatial extent of the lattice that leads to negligible effects from the zero modes [27].

In Fig. 18, we show the matching of the pion mass between the unitary setup and the mixed action setup. We find equal pion masses $\left(m_{\pi}^{\mathrm{tm}}=m_{\pi}^{\text {ov }}\right)$ at around $a m_{\mathrm{ov}}=0.017$. At this matching quark mass, we then compare the value of the pion decay constant. $f_{\pi}^{\text {ov }}$ is greater than $f_{\pi}^{\text {tm }}$ by $7.7 \%$ ( $6.8 \sigma$ away). This difference can be compared to the differences between $f_{\pi}^{\text {tm }}$ and $f_{\pi}^{\text {ov }}$ for other ensembles at the same value of the lattice spacing: $40.0 \%(L \approx 1.3$ $\left.\mathrm{fm}, a \mu=0.004, m_{\pi} L \approx 2.5\right), 27.0 \%\left(L \approx 1.6 \mathrm{fm}, a \mu=0.004, m_{\pi} L \approx 2.8\right)$, $19.5 \%\left(L \approx 2 \mathrm{fm}, a \mu=0.004, m_{\pi} L \approx 3.3\right), 16.5 \%(L \approx 1.3 \mathrm{fm}, a \mu=0.0074$, $\left.m_{\pi} L \approx 3.1\right)$. Indeed, for the current ensemble of interest, the difference of around $7.7 \%$ is much smaller than for other ensembles at $\beta=3.9$, with either a smaller physical volume, or a smaller pion mass (or both). While certainly this can not be treated as a proof that the role of the zero modes is negligible for this ensemble, we believe that this provides a clear hint that our interpretation of the role of the zero modes in our overlap/MTM mixed 
action setup is correct.

\section{References}

[1] H. B. Nielsen and M. Ninomiya, Phys. Lett. B 105 (1981) 219.

[2] M. Lüscher, Phys. Lett. B 428 (1998) 342 [arXiv:hep-lat/9802011].

[3] P. H. Ginsparg and K. G. Wilson, Phys. Rev. D 25 (1982) 2649.

[4] P. Hasenfratz, Nucl. Phys. Proc. Suppl. 63 (1998) 53 [hep-lat/9709110].

[5] P. Hasenfratz, V. Laliena and F. Niedermayer, Phys. Lett. B 427 (1998) 125 [hep-lat/9801021].

[6] P. Hasenfratz, Nucl. Phys. B 525 (1998) 401 [hep-lat/9802007].

[7] F. Niedermayer, Nucl. Phys. Proc. Suppl. 73 (1999) 105 [arXiv:heplat/9810026].

[8] H. Neuberger, Phys. Lett. B 417 (1998) 141 [hep-lat/9707022].

[9] H. Neuberger, Phys. Lett. B 427 (1998) 353 [hep-lat/9801031].

[10] P. Gerhold and K. Jansen, fermions," JHEP 0907 (2009) 025 [arXiv:0902.4135 [hep-lat]].

[11] P. Gerhold and K. Jansen, JHEP 1004 (2010) 094 [arXiv:1002.4336 [hep-lat]].

[12] P. Gerhold, K. Jansen and J. Kallarackal, JHEP 1101 (2011) 143 [arXiv:1011.1648 [hep-lat]].

[13] C. Hoelbling, PoS LATTICE 2010 (2010) 011 [arXiv:1102.0410 [heplat]l.

[14] P. Hernandez, K. Jansen and M. Lüscher, Nucl. Phys. B 552 (1999) 363 [hep-lat/9808010].

[15] C. Allton, C. Maynard, A. Trivini and R. Tweedie, PoS LAT2006 (2006) 202 [arXiv:hep-lat/0610068].

[16] A. Li et al. [xQCD Collaboration], Substitution," Phys. Rev. D 82 (2010) 114501 [arXiv:1005.5424 [hep-lat]]. 
[17] S. Durr et al., PoS LAT2007 (2007) 115 [arXiv:0710.4769 [hep-lat]].

[18] F. Bernardoni, P. Hernandez, N. Garron, S. Necco and C. Pena, Phys. Rev. D 83 (2011) 054503 [arXiv:1008.1870 [hep-lat]].

[19] D. B. Renner et al. [LHP Collaboration], Nucl. Phys. Proc. Suppl. 140, 255 (2005) [arXiv:hep-lat/0409130].

[20] S. R. Beane, P. F. Bedaque, K. Orginos and M. J. Savage, Phys. Rev. D 75 (2007) 094501 [arXiv:hep-lat/0606023].

[21] C. Aubin, J. Laiho and R. S. Van de Water, Phys. Rev. D 77 (2008) 114501 [arXiv:0803.0129 [hep-lat]].

[22] M. Constantinou et al. [ETM Collaboration], Phys. Rev. D 83 (2011) 014505 [arXiv:1009.5606 [hep-lat]].

[23] F. Farchioni, G. Herdoiza, K. Jansen, A. Nube, M. Petschlies and C. Urbach, PoS LATTICE 2010 (2010) 128 [arXiv:1012.0200 [hep-lat]].

[24] G. Herdoiza, PoS LATTICE 2010 (2010) 010 [arXiv:1103.1523 [heplat]].

[25] R. Frezzotti and G. C. Rossi, JHEP 0408 (2004) 007 [arXiv:heplat/0306014].

[26] K. Cichy et al. [ETM Collaboration], PoS LATTICE 2010 (2010) 077 [arXiv:1011.0639 [hep-lat]].

[27] K. Cichy, G. Herdoiza and K. Jansen, Nucl. Phys. B 847 (2011) 179 [arXiv:1012.4412 [hep-lat]].

[28] D. Friedan, Commun. Math. Phys. 85 (1982) 481.

[29] T. Chiarappa et al., arXiv:hep-lat/0609023.

[30] Z. Fodor, S. D. Katz and K. K. Szabo, JHEP 0408 (2004) 003 [arXiv:hep-lat/0311010].

[31] N. Cundy, Nucl. Phys. Proc. Suppl. 153 (2006) 54-61. [heplat/0511047].

[32] S. Schaefer, PoS LAT2006 (2006) 020. [hep-lat/0609063].

[33] N. Cundy, S. Krieg, T. Lippert et al., Comput. Phys. Commun. 180 (2009) 201-208. [arXiv:0803.0294 [hep-lat]]. 
[34] H. Fukaya, S. Hashimoto, K. I. Ishikawa, T. Kaneko, H. Matsufuru, T. Onogi and N. Yamada [JLQCD Collaboration], Phys. Rev. D 74 (2006) 094505 [arXiv:hep-lat/0607020].

[35] S. Aoki et al. [JLQCD Collaboration], Phys. Rev. D 78 (2008) 014508 [arXiv:0803.3197 [hep-lat]].

[36] W. Bietenholz et al. [XLF Collaboration], JHEP 0412 (2004) 044 [arXiv:hep-lat/0411001].

[37] R. Frezzotti, P. A. Grassi, S. Sint and P. Weisz [Alpha collaboration], JHEP 0108 (2001) 058 [arXiv:hep-lat/0101001].

[38] K. Jansen et al. [ XLF Collaboration ], JHEP 0509 (2005) 071. [heplat/0507010].

[39] R. Baron et al. [ ETM Collaboration ], JHEP 1008, 097 (2010). [arXiv:0911.5061 [hep-lat]].

[40] K. Jansen et al. [ XLF Collaboration ], Phys. Lett. B624 (2005) 334341. [hep-lat/0507032].

[41] R. Frezzotti, G. Rossi, PoS LAT2007 (2007) 277. [arXiv:0710.2492 [hep-lat]].

[42] P. Dimopoulos, R. Frezzotti, C. Michael et al., Phys. Rev. D81 (2010) 034509. [arXiv:0908.0451 [hep-lat]].

[43] P. Boucaud et al. [ETM Collaboration], Phys. Lett. B 650 (2007) 304 [arXiv:hep-lat/0701012].

[44] P. Boucaud et al. [ETM collaboration], Comput. Phys. Commun. 179 (2008) 695 [arXiv:0803.0224 [hep-lat]].

[45] R. Baron et al., JHEP 1006 (2010) 111. [arXiv:1004.5284 [hep-lat]].

[46] R. Baron et al. [European Twisted Mass Collaboration], Comput. Phys. Commun. 182 (2011) 299 [arXiv:1005.2042 [hep-lat]].

[47] R. Sommer, Nucl. Phys. B411 (1994) 839-854. [hep-lat/9310022].

[48] M. Constantinou, P. Dimopoulos, R. Frezzotti et al., JHEP 1008 (2010) 068. [arXiv:1004.1115 [hep-lat]].

[49] K. Cichy, G. Herdoiza and K. Jansen, Acta Phys. Polon. Supp. 2 (2009) 497 [arXiv:0910.0816 [hep-lat]]. 
[50] A. Hasenfratz and F. Knechtli, Phys. Rev. D 64 (2001) 034504 [heplat/0103029].

[51] T. Draper, N. Mathur, J. Zhang, A. Alexandru, Y. Chen, S. -J. Dong, I. Horvath and F. X. Lee et al., hep-lat/0609034.

[52] S. Durr, Z. Fodor, C. Hoelbling, S. D. Katz, S. Krieg, T. Kurth, L. Lellouch and T. Lippert et al., JHEP 1108 (2011) 148 [arXiv:1011.2711 [hep-lat]].

[53] C. Alexandrou et al. [ETM Collaboration], Phys. Rev. D 80 (2009) 114503 [arXiv:0910.2419 [hep-lat]].

[54] K. Jansen and M. Schmaltz, Phys. Lett. B 296 (1992) 374 [heplat/9209002].

[55] O. Bär, K. Jansen, S. Schaefer, L. Scorzato and A. Shindler, PoS LAT 2006 (2006) 199 [hep-lat/0609039].

[56] O. Bär, G. Rupak and N. Shoresh, Phys. Rev. D 67 (2003) 114505 [hep-lat/0210050].

[57] O. Bär, G. Rupak, N. Shoresh, Phys. Rev. D70 (2004) 034508. [heplat/0306021].

[58] S. R. Sharpe, J. M. S. Wu, Phys. Rev. D71 (2005) 074501. [heplat/0411021].

[59] G. Münster and C. Schmidt, Europhys. Lett. 66 (2004) 652 [arXiv:heplat/0311032].

[60] L. Scorzato, Eur. Phys. J. C 37 (2004) 445 [arXiv:hep-lat/0407023].

[61] A. Furchner, Unitarity violation in mixed-action lattice QCD, Diploma Thesis (unpublished), Humboldt Universität zu Berlin, http://people.physik.hu-berlin.de/ furchner/Furchner 2010 - Unitarity violation in mixed-action lattice QCD.pdf

[62] J. -W. Chen, D. O'Connell and A. Walker-Loud, Phys. Rev. D 75 (2007) 054501 [hep-lat/0611003].

[63] J. -W. Chen, D. O'Connell and A. Walker-Loud, JHEP 0904 (2009) 090 [arXiv:0706.0035 [hep-lat]].

[64] J. -W. Chen, M. Golterman, D. O'Connell, A. Walker-Loud, Phys. Rev. D79 (2009) 117502. [arXiv:0905.2566 [hep-lat]]. 
[65] O. Bär, M. Golterman and Y. Shamir, Phys. Rev. D 83 (2011) 054501 [arXiv:1012.0987 [hep-lat]].

[66] M. T. Hansen and S. R. Sharpe, Phys. Rev. D 85 (2012) 014503 [arXiv:1111.2404 [hep-lat]].

[67] J. Gasser and H. Leutwyler, Annals Phys. 158 (1984) 142.

[68] K. Orginos and A. Walker-Loud, Phys. Rev. D 77 (2008) 094505 [arXiv:0705.0572 [hep-lat]].

[69] M. Lujan, A. Alexandru, T. Draper, W. Freeman, M. Gong, F. X. Lee, A. Li and K. F. Liu et al., arXiv:1204.6256 [hep-lat].

[70] W. A. Bardeen, A. Duncan, E. Eichten, N. Isgur and H. Thacker, Phys. Rev. D 65 (2001) 014509 [hep-lat/0106008].

[71] W. A. Bardeen, E. Eichten and H. Thacker, Phys. Rev. D 69 (2004) 054502 [hep-lat/0307023].

[72] S. Prelovsek, C. Dawson, T. Izubuchi, K. Orginos and A. Soni, Phys. Rev. D 70, 094503 (2004) [hep-lat/0407037].

[73] M. Golterman, T. Izubuchi, Y. Shamir, Phys. Rev. D71 (2005) 114508. [hep-lat/0504013].

[74] R. Brower, S. Chandrasekharan, J. W. Negele and U. J. Wiese, Phys. Lett. B 560 (2003) 64 [hep-lat/0302005].

[75] G. Herdoiza, K. Jansen, K. Ottnad, C. Urbach, in preparation.

[76] S. R. Sharpe, Phys. Rev. D 74 (2006) 014512 [hep-lat/0606002].

[77] G. Akemann, P. H. Damgaard, K. Splittorff and J. J. M. Verbaarschot, Phys. Rev. D 83 (2011) 085014 [arXiv:1012.0752 [hep-lat]].

[78] K. Splittorff and J. J. M. Verbaarschot, Phys. Rev. D 85 (2012) 105008 [arXiv:1201.1361 [hep-lat]].

[79] C. Alexandrou et al. [European Twisted Mass Collaboration], Phys. Rev. D 78, 014509 (2008) [arXiv:0803.3190 [hep-lat]].

[80] S. Güsken, Nucl. Phys. Proc. Suppl. 17, 361 (1990).

[81] C. Alexandrou, S. Güsken, F. Jegerlehner, K. Schilling and R. Sommer, Nucl. Phys. B 414, 815 (1994) [hep-lat/9211042]. 
[82] M. Albanese et al. [APE Collaboration], Phys. Lett. B 192, 163 (1987).

[83] C. Gattringer et al. [BGR and Bern-Graz-Regensburg Collaborations], Nucl. Phys. B 677, 3 (2004) [hep-lat/0307013]. 